\title{
Cutaneous melanoma
}

\section{Editors:}

\author{
Piotr Rutkowski, Piotr J. Wysocki
}

\section{Authors:}

\section{Piotr Rutkowski $^{1}$, Piotr J. Wysocki ${ }^{2,3}$, Anna Nasierowska-Guttmejer ${ }^{4,5}$, Arkadiusz Jeziorski ${ }^{6}$, Wojciech M. Wysocki ${ }^{7}$, Ewa Kalinka ${ }^{8}$, Tomasz Świtaj', Katarzyna Kozak ${ }^{1}$, Grażyna Kamińska-Winciorek ${ }^{9}$, Anna M. Czarnecka ${ }^{1}$, Hanna Koseła-Paterczyk ${ }^{1}$, Piotr Wiśniewski ${ }^{10}$, Anna Szumera-Ciećkiewicz ${ }^{10,11}$, Marcin Zdzienicki', Bożena Cybulska-Stopa ${ }^{12}$, Marek Ziobro ${ }^{12}$, Jacek Fijuth ${ }^{13}$, Andrzej Kawecki ${ }^{14}$, Lidia Rudnicka $^{15}$, Witold Owczarek ${ }^{16}$, Maciej Krzakowski ${ }^{17}$}

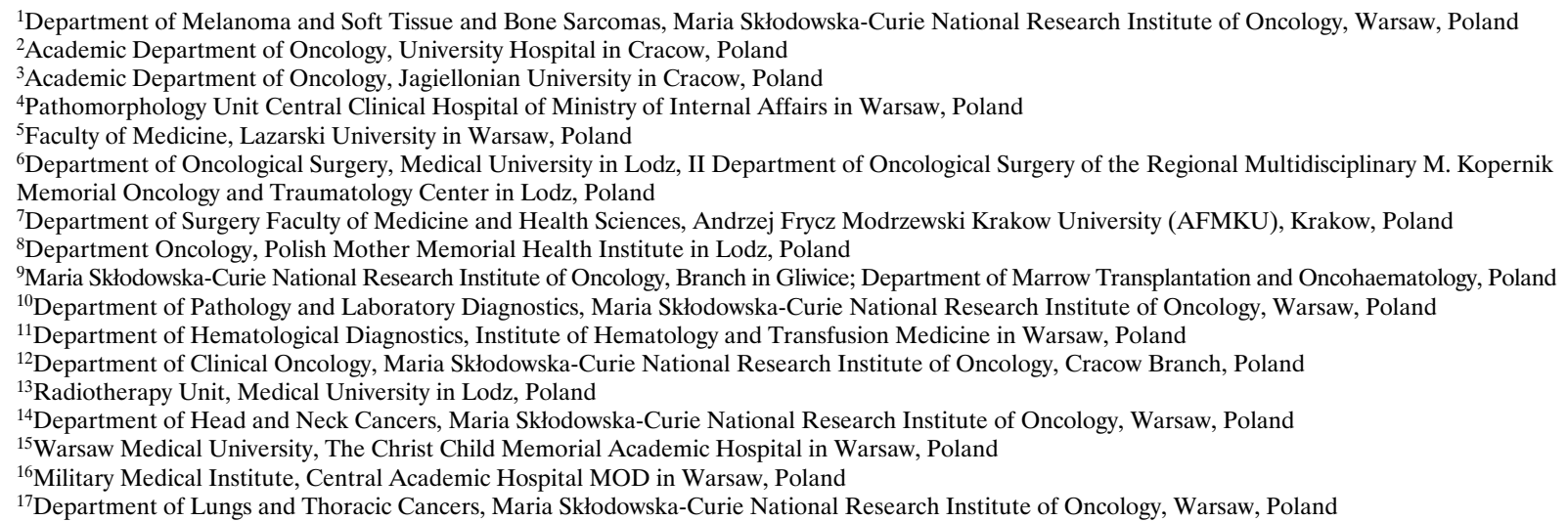

Key words: melanoma, diagnosis, therapy, immunotherapy, anti-PD-1, anti-CTLA-4, BRAF, MEK, sentinel lymph node biopsy

\section{Table of contents}

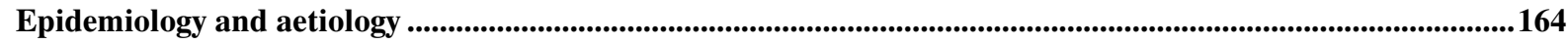

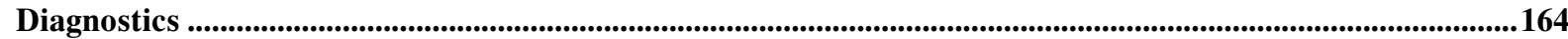

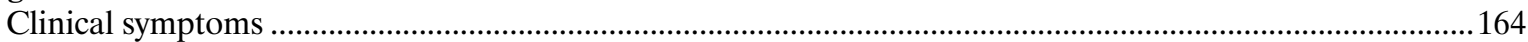

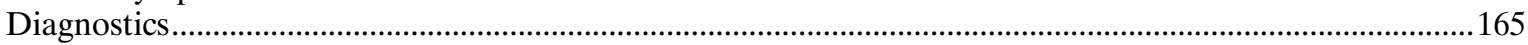

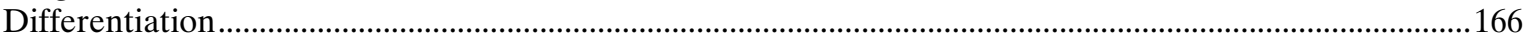

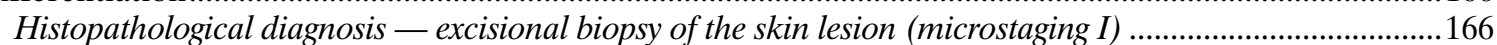

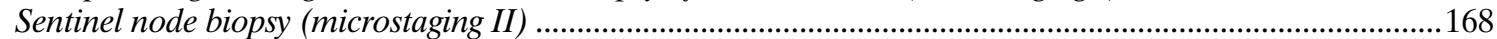

Evaluation of tumour stage and prognostic factors .......................................................................................................169

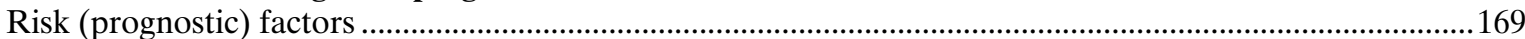

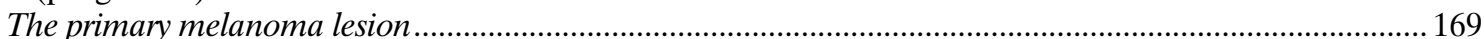

Metastases to the regional lymph nodes (clinical stage III) ..................................................................... 169

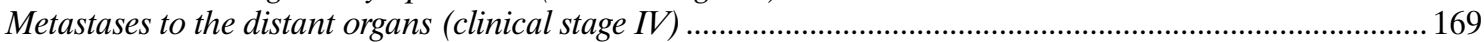

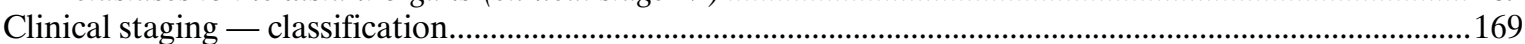

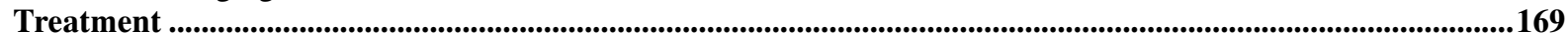

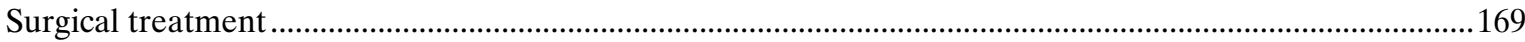

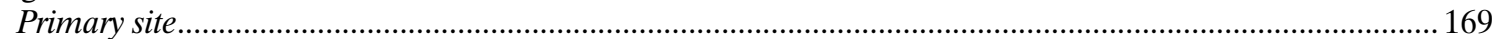

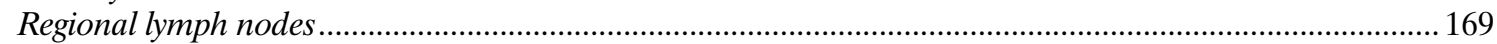

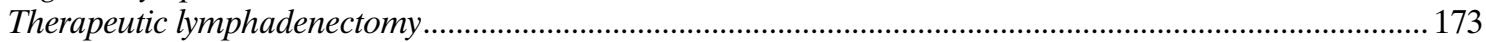

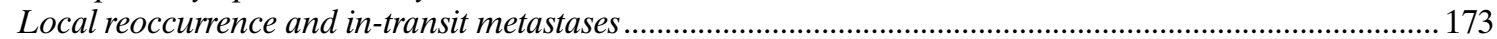

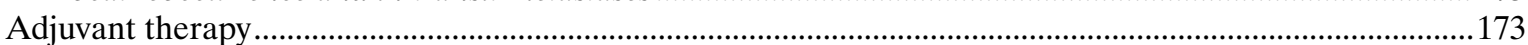

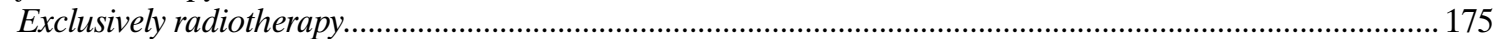

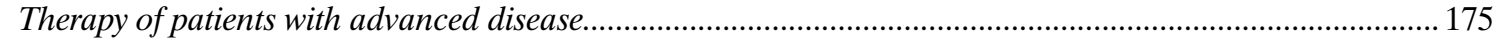

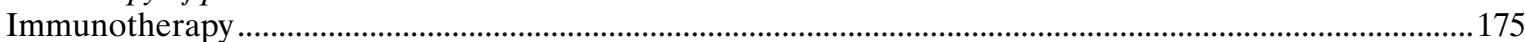

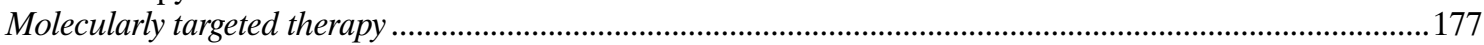




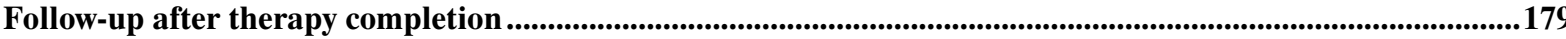

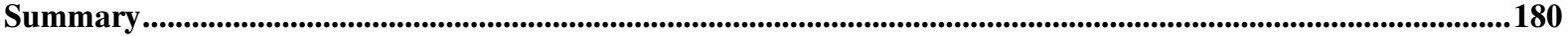

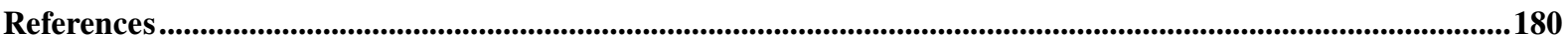

According to the authors and editors, this report contains the most justified principles of diagnostic and therapeutic procedures prepared considering the scientific value of evidence and category of recommendations. These principles should always be interpreted in the context of an individual clinical situation. The recommendations do not always correspond to the current reimbursement rules in Poland. In case of doubt, the current possibilities of reimbursement of individual procedures should be established.

1. The quality of scientific evidence

I - Scientific evidence obtained from well-designed and conducted randomized clinical trials or meta-analyses of randomized clinical trials

II - Scientific evidence obtained from well-designed and conducted prospective observational studies (non-randomized cohort studies)

III - Scientific evidence obtained from retrospective observational studies or case-control studies

$I V$ - Scientific evidence obtained from clinical experiences and/or experts, opinions

2. Category of recommendations

A - Indications confirmed unambiguously and absolutely useful in clinical practice

$B$ - Indications probable and potentially useful indications in clinical practice

$C$ - Indications determined individually

\section{Epidemiology and aetiology}

Cutaneous melanomas are malignant neoplasms deriving from neuroendocrine melanocytic cells. Melanoma are relatively rare in Poland - the standardised incidence rate reaches about $6 / 100,000$, which represents 3800 new melanoma cases per year during the last few years (about 1800 men and about 2000 women). However, the incidence rate of melanoma is increasing rapidly compared to other neoplasms. A threefold increase of melanoma morbidity has been observed in Poland during the years 1980 to 2010. The median age at diagnosis is similar for both sexes and equals about 50 years. The standardised mortality rate reaches 2.1/100,000 men and 1.4/100,000 women, which represents, during the last years, respectively, about 700 and 710 melanoma-related deaths [1-3].

The influence of the natural ultraviolet radiation (solar rays) and artificial radiation (e.g. tanning beds, solarium), permanent mechanical or chemical irritation, low content of pigment in the skin, and genetic predispositions (e.g. familial atypical mole syndrome; FAMS) constitute risk factors of melanomas (III, B). Protection against excessive action of ultraviolet light is the most important element of primary melanoma prophylaxis (III, A).

Cutaneous melanoma has a unique chance to be cured due to its localisation, which enables early identification of the primary site (microstaging I - excisional biopsy of the primary lesion) and of the metastases to the locoregional lymph nodes (microstaging II - sentinel nodes biopsy).

In about $80 \%$ of patients, cutaneous melanoma is a limited, localised disease, while a loco-regional advanced or metastatic disease is primarily diagnosed in, respectively, $15 \%$ and $5 \%$ of patients. Progress in the adjuvant and palliative therapy of patients with metastatic melanoma is still unsatisfactory. The five-year overall survival rates reach in early stages of melanoma $70-95 \%$ as well as $20-70 \%$ and $20-30 \%$ in regionally advanced and metastatic disease respectively with the use of modern systemic therapy.

The crucial recommendation is to treat a melanoma patient with a multidisciplinary team formed by specialists experienced in diagnosing and treating melanoma - this in particular concerns patients with stage III and IV melanomas [4, 5].

\section{Diagnostics}

\section{Clinical symptoms}

Skin melanomas may be suspected in both de novo skin changes and in alterations of pre-existing moles. There have been some attempts to create diagnostic systems based on clinical symptoms (Table 1). The most popular of these is the American mnemonic clinical system called $\mathrm{ABCD}(\mathrm{E})$, used mostly with educational intent because it is useful only in identification of some melanomas, mostly of the superficial spreading melanomas and the majority of advanced melanomas. However, this system cannot be used as a diagnostic (screening) tool in daily clinical practice. A clinical $\mathrm{ABCD}(\mathrm{E})$ system does not permit appropriate qualification of about $50 \%$ of melanomas (especially including the early stages of skin melanomas with diameter $<5 \mathrm{~mm}$, nodular melanoma usually without parameter $\mathrm{C}$ - heterogeneity of colour and $\mathrm{B}$ - irregular border as well as amelanotic melanomas and changes of the hairy skin of the head surface) [1].

Thin melanomas $(<1 \mathrm{~mm}$ of thickness according to Breslow scale) are usually identified during the medical examination, whereas very rarely by the patient their relatives. 
Table 1. American $A B C D(E)$ system, which enables the initial identification of a part of melanomas based on a clinical examination without use of any supplementary diagnostic methods

ABCD system

A - asymmetry (a melanoma, in contrast to usually round or ellipsoidal benign changes, is asymmetrical in relation to any axis. Melanoma presents as an uneven change composed of elevations called 'islands')

B - borders (irregular and unravelled)

C - colour (the presence of more than one colour [from bright brown to black or steel blue] or the uneven distribution of colour, often with spotted distribution of the pigment [especially visible on the dermatoscopy])

D - diameter (diameter $>5 \mathrm{~mm}$ or dynamics of the morphological sizes in a tumour)

E - elevation or evolution (elevation of surface over the level of the change surrounding epidermis. Thin melanomas

[thickness $\leq 1 \mathrm{~mm}$ according to Breslow scale] do not form a palpable node compared to a normal skin surrounding the lesion; increase of the diameter [extension or evolution] of the primary change is more significant than its elevation)

\section{Diagnostics}

Medical history should include questions concerning skin condition (information concerning changes of the pre-existing skin moles, the appearance of new pigmentary lesions, and accompanying symptoms, e.g. pruritus) and risk factors of cutaneous melanoma (e.g. sunburn, use of tanning beds, melanomas in relatives, and previous immunosuppressive treatment or HIV infection). It is important to stress that in more than $60 \%$ of melanoma diagnosed after physical examination patients did not report any specific data in anamnesis, which can be helpful to establish this diagnosis.

We should stress that whole skin examination is a crucial method of detecting skin melanomas and should be performed by each physician during the ambulatory visit or hospitalisation of any patient. The major rule of the visual inspection is to evaluate the total skin surface in appropriate lighting, also including the hard-to-reach areas (head, feet, interdigital spaces, urogenital, and perianal areas).

The recommended test, used in preliminary, quick, non-invasive diagnostics, is dermoscopy (dermatoscopy) (II, A) [6, 7]. The examination consists of assessment of all lesions on the patient's skin by means of a manual dermoscope with polarised or non-polarised light with $10 \times$ magnification [7]. Thanks to dermoscopy it is possible to improve the diagnostic sensitivity by about $30 \%$. The simplest technique of dermoscopic assessment (the so-called three-point dermoscopic scale according to Argenziano) is based on the clinical suspicion of melanoma when two of the following three criteria are met: 1) asymmetric distribution of the dermoscopic structures within the change, 2) atypical pigmentation network, and 3) blue-white veil. The sensitivity of this diagnostic method reaches $96.3 \%$ and specificity $94.2 \%$. Other methods of dermatoscopic analysis including the dermatoscopic method $\mathrm{ABCD}$, pattern analysis, seven-point scale, Menzies's method, or CASH (colour, architecture, symmetry, homogeneity) algorithm are characterised by similar sensitivity and slightly higher specificity. It should be stressed that the presented dermatoscopic evaluation systems cannot be used to assess lesions placed in 'special locations' including changes of palms and soles of the feet, the hairy skin of the head surface, the skin of the face, mucosa of the mouth, and the external sex organs. In such cases it is necessary to apply dermatoscopic algorithms, dedicated to the character of the skin of each localisation. In the case of atypical mole syndrome, it may be useful to collect photographic records of a lesion or of the total skin surface (total body photography) and to compare taken pictures and observed skin lesions in consecutive time sequences. There are some systems that automatically compare dermatoscopic pictures taken in different time sequences; however, they are not commonly used due to their technological limitations.

An initial dermatoscopic diagnosis may by verified by use of the confocal reflection microscopy (in the scope of a specialist dermatological consultation). In some justified cases when an excisional biopsy cannot be performed (e.g. when melanoma is suspected in the area of the extensive congenital moles in small children), it is possible to perform a dermatoscopy-guided biopsy in order to obtain a sample for further histopathological examination.

Histopathological examination of the whole excised mole is crucial for diagnosing a skin melanoma. Procedures other than excisional biopsy (microstaging I) do not permit an appropriate diagnosis (III, A).

Once a histopathological diagnosis of a skin melanoma has been made a clinical stage tailored therapy should be implemented (see below).

The supplementary diagnostic tests used in clinical staging of the melanoma include: essential blood test [peripheral blood morphology, liver enzymes levels, lactate dehydrogenase (LDH) activity], radiologic exam (RTG) of the chest in an anteroposterior and in lateral projection, as well as the ultrasonographic exam of the abdomen and of the locoregional lymph nodes. First of all, a thorough physical examination should be carried out, including the examination of the whole skin (presence of other suspicious pigmented lesions, satellite and/or in transit changes), assessment of lymph nodes, and examination for the presence of possible distant metastasis. In low-risk clinical melanomas (pT1a), other tests are not routinely required. 
Table 2. Clinical differential diagnostics ofcutaneous melanoma

\begin{tabular}{|c|c|}
\hline Early skin melanoma & $\begin{array}{l}\text { - Pigmented naevus, including junction naevus (naevus melanocyticus junctionalis, marginalis) } \\
\text { and compound nevus (naevus melanocyticus compositus) } \\
\text { - Blue nevus (naevus coeruleus) } \\
\text { - Simple lentigo (lentigo simplex) } \\
\text { - Actinic keratosis or solar keratosis } \\
\text { - Superficial basal cell carcinoma (carcinoma basocellulare superficiale) } \\
\text { - Spitz's naevus } \\
\text { - Tattoo }\end{array}$ \\
\hline $\begin{array}{l}\text { Locally advanced } \\
\text { melanoma }\end{array}$ & $\begin{array}{l}\text { - Seborrheic keratosis (verruca seborrhoica, keratosis seborrhoica) } \\
\text { - Dermatofibroma } \\
\text { - Keratoacanthoma } \\
\text { - Pigmented basal cell carcinoma (carcinoma basocellulare pigmentosum) } \\
\text { - Haemangioma } \\
\text { - Venous extravasation } \\
\text { - Pyogenic granuloma (granuloma pyogenicum) and telangiectatic granuloma } \\
\quad \text { (granuloma telangiectaticum) } \\
\text { - Pigmented hidrocystoma } \\
\text { - Kaposi's sarcoma } \\
\text { - Angiomyoneuroma } \\
\text { - Other adnexal tumours, especially pigmented } \\
\text { - Onychomycosis } \\
\text { - Subungual or under cutaneous corn haemangioma }\end{array}$ \\
\hline
\end{tabular}

However, in higher stages (pT1b-pT4b), a scan should be performed by ultrasound examination of regional lymph nodes, and a suspected biopsy should be performed with a histological evaluation before the scar is removed and the sentinel node biopsy is performed. In patients without symptoms, there is no need to perform other additional tests, which mainly concerns computed tomography of the brain, chest, abdominal cavity and pelvis with contrast (CT) and positron emission tomography (PET-CT) (IV, A). CT or PET-CT may be considered in patients with diagnosed skin melanoma in clinical stage IIC and III (PET-CT if the clinical metastases to the lymph nodes are present) or with isolated metastases to the distant organs (potentially resectable). In the case of the clinical metastases to the inguinal lymph nodes it is recommended that CT or magnetic resonance imaging (MRI) of the pelvis and abdomen be performed.

In patients with melanoma metastases from an unknown primary site to the lymph nodes or to the skin, a primary lesion should be searched for carefully (especially on the hairy skin of the head surface and the mucosal membranes) and a detailed medical history taken (e.g. concerning any cosmetic medicine ablation methods applied to any lesion), in such a clinical situation other imaging tests are recommended (CT or PET-CT of brain, neck, chest, abdominal cavity, pelvis) (IV, B).

\section{Differentiation}

The conditions that should be considered in the differential diagnostics of early and locally advanced skin melanoma are presented in Table 2.

\section{Histopathological diagnosis - excisional biopsy of the skin lesion (microstaging I)}

An excisional biopsy of the clinically suspected skin lesion is a method of choice because it allows confirmation of a microscopic diagnosis of melanoma and collection of data concerning the crucial risk factors, crucial for planning a further therapeutic approach (microstaging) (III, A) [1, 5, 8]. There are no indications for prophylactic excision of skin moles that are not suspected of being skin melanoma.

Pathomorphological examination of samples from the excisional biopsy consists of macro- and microscopic assessment of all elements which should contain a standardized histopathological report (http:/www.pol-pat. $\mathrm{pl} /$ pliki/files/standardy_pdf/1.2_czerniak.pdf):

1. Macroscopic assessment

a. Size of the excised skin section with the lesion (three dimensions);

b. Size of the lesion (two dimensions);

c. Pigmentation (homogenous, heterogeneous);

d. Border of the lesion (regular, irregular);

e. Nodule (present, not present);

f. Margins (lateral and deep margin).

2. Microscopic assessment

Microscopic features/characteristics that are required:

a. Breslow thickness of infiltration (in millimetres) is measured from the top of the granular layer of the epidermis, or if the surface is ulcerated - from the base of the ulcer, to the deepest invasive cell across the broad base of the tumour;

b. Tumour stage pT; 
c. Presence or absence of ulceration including the whole thickness of the epidermis covering the tumour as well as information about the extent of ulceration, measured either as the diameter or percentage of tumour width;

d. Mitotic count per square millimetre of the invasive melanoma (only in a vertical component, in the mitotic high-power fields that equates to $1 \mathrm{~mm}^{2}$, so-called hot spots);

e. Growth phases (horizontal [radial] — intraepidermal, in situ with microinvasion and sagittal [vertical], always skin invasion);

f. Presence or absence of microscopic satellite sites (sites composed of melanocytes with diameter $>0.05 \mathrm{~mm}$ remoted $>0.3 \mathrm{~mm}$ and $<2 \mathrm{~cm}$ from the invasive component of the primary melanoma tumour - parameter $\mathrm{N}$ ).

g. Peripheral margin (measured from the in situ to the invasive component) and in depth;

Recommended elements:

h. Presence and extend of tumour regression;

i. Clark level of invasion (level I, II, III, IV, V);

j. Histopathological subtype (see below: WHO histopathological classification of skin tumors World Health Organization; [WHO]);

k. Cell type (epithelioid, fusiform, small, pleomorphic, other);

1. Presence and grading of the lymphocytic infiltration (tumour infiltrating lymphocytes [TILs]; evaluated only in a vertical component; absent, moderate - TILs non-brisk, abundant - TILS brisk);

m. Presence or absence of lymph and blood vessel infiltration;

n. Presence or absence of nerve trunk infiltration;

o. Presence of a mole.

WHO classification of skin tumours $4^{\text {th }}$ Edition

2018 distinguishes the following types of melanoma [9]:

- melanocytic tumours in intermittently sun-exposed skin;

- superficial spreading melanoma, low-SCD melanoma);

- melanocytic tumours in chronically sun-exposed skin;

- lentigo maligna melanoma;

- desmoplastic melanoma;

- Spitz melanoma;

- acral melanoma;

- mucosal melanoma;

- mucosal lentiginous melanoma;

- mucosal nodular melanoma;

- melanoma arising in blue naevus;

- melanoma arising in giant congenital naevus;

- ocular melanocytic tumours;

- uveal melanoma (epithelioid cell melanoma, spindle cell melanoma type A, spindle cell melanoma type B);

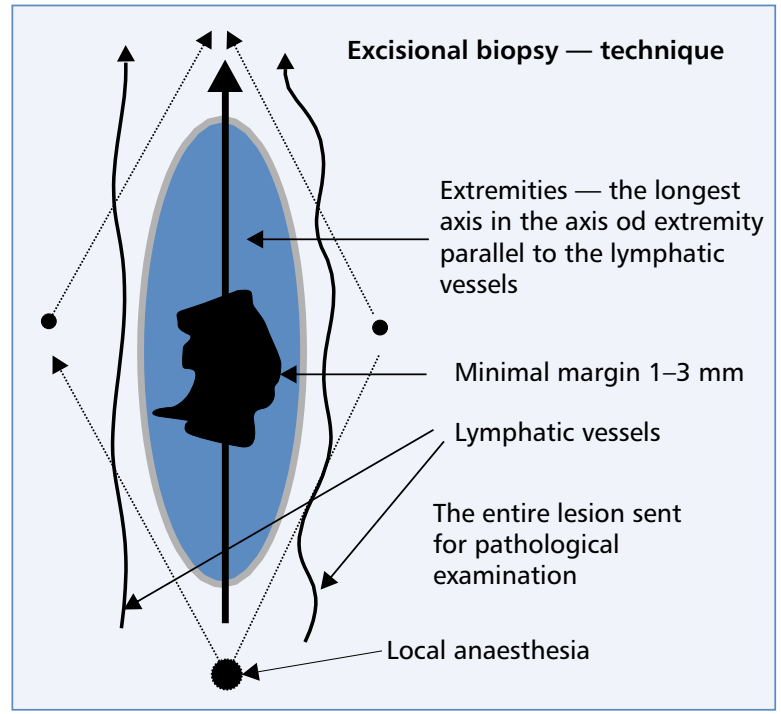

Figure 1. (According to W. Ruka) Recommended direction of the cut during the excisional biopsy. Spindle-shaped excision of the suspected pigmentary lesion should be made collaterally to the regional lymph vessel (toward the nearest draining lymph node/lymph vessel confluence), in the majority of cases enabling a primary suture of the wound

- conjunctival melanoma;

- nodular melanoma;

- naevoid melanoma;

- metastatic melanoma.

An excisional biopsy is a simple surgical procedure that can usually be performed in an outpatient clinic. Excision of the suspected skin change is done in local infiltration anaesthesia. The lateral excision margin should include 1-3 mm of healthy skin. The surgical specimen should include not only the whole thickness of the skin but also a superficial layer of the adipose tissue. The fascia should not be excised, and the wound should be closed by a primary suture. The skin should be cut as an ellipse specimen following the lines of relaxed skin tension (Fig. 1). Only the cut of the face lesion should follow the aesthetic lines. Transversal cuts should never be done (on the limb area) because in the case of repeated surgery they give a poor cosmetic effect and are inconsistent with oncological recommendations.

Results of fine- or core-needle aspiration biopsy or of the incisional (section) or shave biopsy do not deliver reliable data (according to recommendations of the American Joint Cancer Committee/Union International Contre le Cancer [AJCC/UICC]) concerning the primary melanoma lesion and therefore should not be used.

If the lesion is extensive and ulcerated, imprint cytology may be performed in order to obtain a sample for cytological examination (a glass slide should be pressed onto the tumour surface and then the material should be referred to cytological examination). 
It is currently known that some defined subtypes of melanoma are associated with specific mutations (e.g. $K I T$ gene mutations - subungual melanoma or mucosal melanomas, in the GNAQ, GNA11 genes - melanomas derived from the blue naevus and the eye). In patients with disseminated (primary or secondary) melanoma, testing for $B R A F$ gene mutation in the FFPE is obligatory as well as in the case of high risk of relapse of melanoma (clinical stage IIIA > $1 \mathrm{~mm}$, IIIB, IIIC and IIID) qualified for systemic therapy and for KIT and NRAS mutation is optional $(\mathrm{V}, \mathrm{A})$. There is no need for repeated sampling of the metastases to detect the presence of molecular disorders. Genetic tests should be performed in referral centres that undergo quality audits. It is not recommended that mutations are tested for inpatients with skin melanoma and no metastatic sites [5].

\section{Sentinel node biopsy (microstaging II)}

A sentinel node biopsy should be done in patients (II, A) $[1,5,10,11]$ :

- after an excisional biopsy and with histopathological confirmation of skin melanoma but not after a wide local excision of a primary site;

— with Breslow thickness $\geq 0.8 \mathrm{~mm}$ or with (micro-) ulceration on the melanoma surface independently of the thickness of the infiltration (melanoma with primary site that has been classified as pT1b-T4b according to TNM AJCC/UICC 2017 classification); according to recommendations of the American Society of Surgical Oncology (SSO), the American Society of Clinical Oncology, and the European Society of Medical Oncology (ESMO), a sentinel node biopsy may be considered in melanoma $\mathrm{pT} 1 \mathrm{~b}$ and thickness $0.8-1.0 \mathrm{~mm}$ and coexistence of additional risk factors, e.g. mitotic index $\geq 1 / \mathrm{mm}^{2}$ (III, A);

- without clinical symptoms of metastases to the regional lymph nodes or to the distant organs.

A sentinel node biopsy is obligatory to assess the presence of micrometastases in the lymph nodes [12]. During the sentinel node biopsy, a preoperative lymphoscintigraphy and a intraoperative lymphoscintigraphy combined with staining should be done. A sentinel node biopsy should be performed after the excisional biopsy of melanoma, simultaneously with radical, wide local excision of the scar after the primary excisional biopsy of melanoma. Accessible data do not indicate any negative prognostic impact of performing the sentinel node biopsy six weeks after the excision of the primary melanoma site (III, B). The accuracy of this method depends on the cooperation of a nuclear medicine specialist, surgeon, and pathologist. A sentinel node biopsy is a diagnostic procedure that is 'minimally invasive' due to low frequency of early and late complications.

All detected lymph nodes should undergo pathophysiological assessment. If the metastatic deposits are macroscopically visible, it is enough to exam only one section, while in all other cases serial sections of the lymph node at every $2-4 \mathrm{~mm}$ should be done. A histopathological report describing this material should include the number of lymph nodes found, the number of lymph nodes with metastases, the size and localisation of the biggest metastatic site, the presence or absence of the extracapsular spreading, and the presence of embolisms of tumour cells in blood vessels. Immunohistochemical exam with use of specific markers (e.g. S100, SOX-10, HMB45, Melan-A) may visualise tiny conglomerates of the neoplastic cells.

The results of the prospective study Multicentre Selective Lymphadenectomy Trial 1 (MSLT-1) suggest that a sentinel node biopsy melanoma helps to identify patients with high risk of metastases, helps to assess the clinical stage of the disease, ensures excellent local disease control, and enables qualification of patients to clinical trials with the use of homogenous criteria [10]. In the MSLT- 1 trial in the whole analysed population of patients who underwent sentinel node biopsy, no disease-free survival time and no overall survival time improvement was proven, compared to the whole study population. However, in a subgroup of patients with present metastases to lymph nodes the overall 10-year survival rate was significantly better in patients in whom an immediate lymphadenectomy had been performed in the case of a positive sentinel node, compared to patients who had received this therapy later for clinically overt metastases (62.1\% vs. $41.5 \%$; $p=0.006)$ [10].

If the histopathological assessment affirms the presence of melanoma metastases to sentinel nodes, a radical lymphadenectomy may be considered (so-called completion lymph node dissection, CLND) because the melanoma metastases to other lymph nodes are detected by routine histopathological methods in about $20-30 \%$ of patients [13] (especially when micrometastasis size exceeds $1 \mathrm{~mm}$ ). An alternative option is an observation with use of ultrasonographic monitoring of the regional lymphatic basin every 4-6 months. The results of two published trials with random selection of patients [14, 15], one of which, however, had insufficient statistical power [15], did not show an improvement in melanoma-dependent overall survival in patients after CLND [14] nor of time to occurrence of distant metastases [15], but progression-free survival in persons after CLND was longer (fewer relapses in the nodal area). These trials also confirmed the basic prognostic role of a sentinel node biopsy (I, B). At present in clinical practice CLND is only performed in patients at high risk of metastases in nonsentinel lymph nodes (such as large size of metastasis to the sentinel node, metastases in $>2$ sentinel nodes or extracapsular infiltration of the sentinel node).

There are ongoing clinical studies evaluating if the adjuvant lymphadenectomy may be limited in some 
patients (sub-micrometastases to the sentinel lymph node with diameter $<0.1 \mathrm{~mm}$ or placed subcapsular and with diameter $<0.4 \mathrm{~mm}$ ) with no negative impact on the melanoma reoccurrence rate [16].

\section{Evaluation of tumour stage and prognostic factors}

Identification of the clinical and pathomorphological risk factors is aimed at understanding the biology of the neoplasm and planning a tailored therapy for a given patient, which considers relapse risk factors and overall survival probability.

\section{Risk (prognostic) factors}

\section{The primary melanoma lesion}

The most important risk factors in patients with skin melanomas without metastases are thickness (Breslow) and the presence of micro(ulceration) of the primary site. An important prognostic value of mitotic index and microsatellitosis as part of parameter $\mathrm{N}$ has recently been proven. These factors are included in TNM system version 8 (Table 3 ) $[5,8,12,17]$.

Metastases to the regional lymph nodes (clinical stage III)

The presence of metastases in the regional lymph nodes is the most important prognostic factor in patients with skin melanomas. In the case of the presence of metastases, the number of involved regional lymph nodes constitutes the principal risk factor. The type of metastases also influences the risk; patients with clinically occult lymph node metastases have better prognosis (neoplastic sites detected during the microscopic exam in the clinically not enlarged and not palpable lymph nodes - excised during the sentinel node biopsy) than patients with clinically apparent lymph node metastases (foci of neoplasm diagnosed during the microscopic exam of regional lymph nodes that are clinically enlarged and palpable, or visible on imaging studies). Extracapsular infiltration of the neoplastic cells constitutes an additional negative risk factor in patients with metastases to the lymph nodes.

\section{Metastases to the distant organs (clinical stage IV)}

Localisation of metastases and LDH activity are the major prognostic factors in patients with extranodal metastases. The worst prognosis in this group of patients is with metastases to the central nervous system.

\section{Clinical staging - classification}

The current TNM classification system for the clinical and pathological staging of cutaneous melanoma comes from the 2017 revision (Table 3) (II, A) [17].

\section{Treatment}

Surgery is a treatment by choice in patients with melanoma (I, A). After performing an excisional biopsy of the suspected pigmented lesion and making a diagnosis of melanoma, we should consider a wide scar excision with appropriate margins and a sentinel node biopsy (Figure 2). In the case of detecting a metastasis in clinically palpable reginal lymph nodes by fine-needle biopsy, lymphadenectomy of the regional lymph nodes should be performed. Lymphadenectomy should be considered if a sentinel node biopsy confirms metastases. In fact, adjuvant therapy after surgery is a standard procedure, and in patients with metastatic disease it should be tailored to the clinical situation. The essential and obligatory recommendation is to refer patients to a multidisciplinary team of specialists experienced in diagnostics and treating melanomas.

\section{Surgical treatment}

\section{Primary site}

Radical therapy of the primary site of melanoma includes a radical wide excision of the scar after the excisional biopsy of the primary site.

Based on the results of six multicentre, randomised trials it was decided to derogate from extended excisions of the primary melanoma site (with margin $\geq 3 \mathrm{~cm}$ ) in favour of narrower margins of healthy tissues. The following are the current recommended margins of radical therapy of the primary melanoma lesion (excision of the scare after excisional biopsy of the primary site): melanoma in situ - margin $5 \mathrm{~mm}$, melanoma with tumour depth $\leq 2 \mathrm{~mm}-\operatorname{margin} 1 \mathrm{~cm}$, and melanoma with tumour depth $>2 \mathrm{~mm}-$ margin $2 \mathrm{~cm}$ (Table 4) (II, A).

Applying margins wider than $2 \mathrm{~cm}$ decreases the local reoccurrence rate but does not improve long-term survival. The scar after an excisional biopsy of a melanoma $\leq 2 \mathrm{~mm}$ should be removed without superficial fascia. These rules cannot be applied for melanomas located on the face, where no fascia is present and the excision margin may be narrower. In the case of the subungual localisation of melanomas, a distant phalanx should be amputated.

\section{Regional lymph nodes}

Patients with melanoma with metastases to the regional lymph nodes are a heterogenous group of patients considering the prognosis (five-year survival range: $15-70 \%$ ). Prospective clinical trials did not confirm any benefit of performing an elective lymphadenectomy in patients without clinical signs of melanoma metastases to the lymph nodes. Currently, lymphadenectomy in patients with cutaneous melanomas is performed only in the case of metastases on 
Table 3. Clinical staging classification according to TNM AJCC/UICC from the year 2017

\section{A. TNM system categories}

\begin{tabular}{|c|c|c|}
\hline Parameter T & Breslow thickness [mm] & (Micro-)ulceration \\
\hline \multicolumn{3}{|l|}{ pTis (in situ) } \\
\hline $\begin{array}{l}\text { Tx the thickness of } \\
\text { the infiltrate cannot } \\
\text { be determined } \\
\text { (e.g. diagnosis } \\
\text { by curettage) } \\
\text { T0 no primary } \\
\text { tumor present } \\
\text { (e.g. unknown } \\
\text { primary site or } \\
\text { complete regression } \\
\text { of primary tumour) }\end{array}$ & Not applicable & Not applicable \\
\hline T1 & $\leq 1.0$ & \\
\hline T1a & $<0.8$ & Without ulceration \\
\hline \multirow{2}{*}{$\mathrm{T} 1 \mathrm{~b}$} & $<0.8$ & With ulceration \\
\hline & $0.8-1.0$ & With or without ulceration \\
\hline $\mathrm{T} 2$ & $>1.0-2.00$ & Unknown or undetermined \\
\hline $\mathrm{T} 2 \mathrm{a}$ & & a) Without ulceration \\
\hline $\mathrm{T} 2 \mathrm{~b}$ & & b) With ulceration \\
\hline T3 & $>2.0-4.0$ & Unknown or undetermined \\
\hline ТЗа & & a) Without ulceration \\
\hline T3b & & b) With ulceration \\
\hline T4 & $>4.0$ & Unknown or undetermined \\
\hline T4a & & a) Without ulceration \\
\hline $\mathrm{T} 4 \mathrm{~b}$ & & b) With ulceration \\
\hline Parameter $\mathbf{N}$ & Number of the regional lymph nodes with metastases & $\begin{array}{l}\text { Presence of an in-transit } \\
\text { metastasis, satellite sites and/or } \\
\text { microsatellite* }\end{array}$ \\
\hline $\mathrm{Nx}$ & $\begin{array}{l}\text { The status of the regional lymph nodes cannot be assessed (e.g. } \\
\text { sentinel node biopsy was not performed or lymph nodes previously } \\
\text { removed for other reasons). Exception: Pathological N category is not } \\
\text { required for grade } \mathrm{T} 1 ; \mathrm{cN} \text { should be used }\end{array}$ & No \\
\hline NO & No metastasis to regional lymph nodes & No \\
\hline N1 & $\begin{array}{l}\text { One lymph node with metastatic transformation or presence of } \\
\text { in-transit metastases satellite and/or microsatellite foci without } \\
\text { involvement of the lymph nodes }\end{array}$ & \\
\hline N1a & $\begin{array}{l}\text { Clinically occult metastasis to one lymph node (detected by sentinel } \\
\text { node biopsy) }\end{array}$ & No \\
\hline $\mathrm{N} 1 \mathrm{~b}$ & Metastasis to one lymph node assessed by clinical exam & No \\
\hline N1c & No metastases to regional lymph nodes & Yes \\
\hline N2 & $\begin{array}{l}\text { Metastases to } 2 \text { or } 3 \text { lymph nodes or presence of in-transit } \\
\text { metastases, satellite and/or microsatellite lesions with simultaneous } \\
\text { metastasis to one lymph node }\end{array}$ & \\
\hline $\mathrm{N} 2 \mathrm{a}$ & $\begin{array}{l}\text { Clinically occult metastases to } 2 \text { or } 3 \text { lymph nodes (detected by } \\
\text { sentinel node biopsy) }\end{array}$ & No \\
\hline $\mathrm{N} 2 \mathrm{~b}$ & Metastases to 2 or 3 lymph nodes, at least one clinically involved & No \\
\hline $\mathrm{N} 2 \mathrm{c}$ & $\begin{array}{l}\text { Metastasis to } 1 \text { lymph node (assessed by sentinel lymph node biopsy } \\
\text { or clinically) }\end{array}$ & Yes \\
\hline N3 & $\begin{array}{l}\text { Metastases to } 4 \text { or more lymph nodes or presence of in-transit, } \\
\text { satellite and/or microsatellite metastases with simultaneous } \\
\text { metastasis to } 2 \text { or more lymph nodes or presence of matted nodes } \\
\text { with or without in-transit, satellite and/or microsatellite metastases }\end{array}$ & \\
\hline N3a & $\begin{array}{l}\text { Clinically occult metastases to } 4 \text { or more lymph nodes (detected by } \\
\text { sentinel node biopsy) }\end{array}$ & No \\
\hline N3b & $\begin{array}{l}\text { Metastases to at least } 4 \text { lymph nodes and at least one as clinically } \\
\text { overt or conglomerate of lymph nodes }\end{array}$ & No \\
\hline $\mathrm{N} 3 \mathrm{c}$ & $\begin{array}{l}\text { Metastases to } 2 \text { or more lymph nodes and/or conglomerate of lymph } \\
\text { nodes }\end{array}$ & Yes \\
\hline
\end{tabular}


Table 3 (cont.). Clinical staging classification according to TNM AJCC/UICC from the year 2017

\begin{tabular}{lll}
\hline Parameter $\mathbf{M}$ & Localisation of the metastases & Serum LDH activity \\
\hline M0 & Without distant metastases & \\
\hline $\begin{array}{l}\text { M1a } \\
\text { M1 } a(0)\end{array}$ & Skin, subcutaneous tissue, or non-regional lymph nodes & Normal \\
M1a(1) & & Increased \\
\hline M1b & Lungs \pm localisations M1a & Normal \\
$M 1 b(0)$ & & Increased \\
$M 1 b(1)$ & Other than above mentioned visceral organs with exclusion of & \\
\hline M1c & central nervous system and \pm localisations M1a and M1b & Normal \\
$M 1 c(0)$ & & Increased \\
$M 1 c(1)$ & & \\
\hline M1d & Metastases to the central nervous system \pm localisations M1a, & \\
$M 1 d(0)$ & M1b or M1c & Normal \\
$M 1 d(1)$ & & Increased \\
\hline
\end{tabular}

*Micro-/satellitosis - neoplastic infiltration or nodules (macro or microscopic) remoted up to $2 \mathrm{~cm}$ from the primary site of the skin melanoma to the level of the nearest regional lymph confluence/drainage; LDH — lactate dehydrogenase

\section{B. Clinical stages}

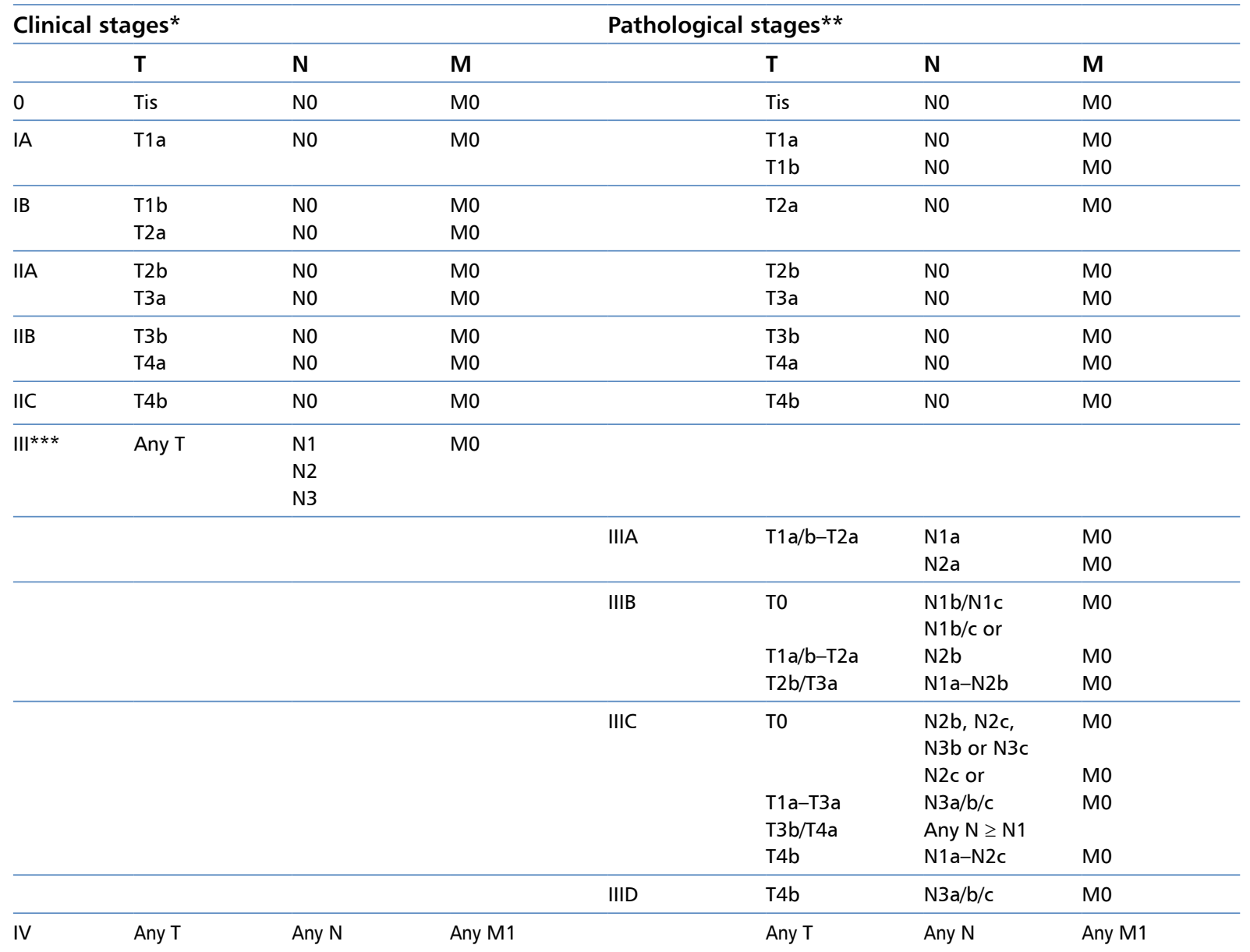

${ }^{*}$ Clinical staging includes micrograding of the primary site and a clinical/radiological/histopathological assessment of presence of metastases. Consequently, clinical staging may be applied only after complete excision of the primary site of the skin melanoma (excisional biopsy) and evaluation of the regional lymph nodes and distant organs for the presence of metastases; ** pathologic grading/staging includes micrograding of the primary site and a pathological assessment of the regional lymph nodes: after a sentinel lymph node biopsy or after a radical lymphadenectomy (except from stage 0 and IA-pTis/pT1 cN0 cM0 in which no procedure is applied to the regional lymph nodes); ${ }^{* * *}$ clinical staging does not include any subgroups of stage III 


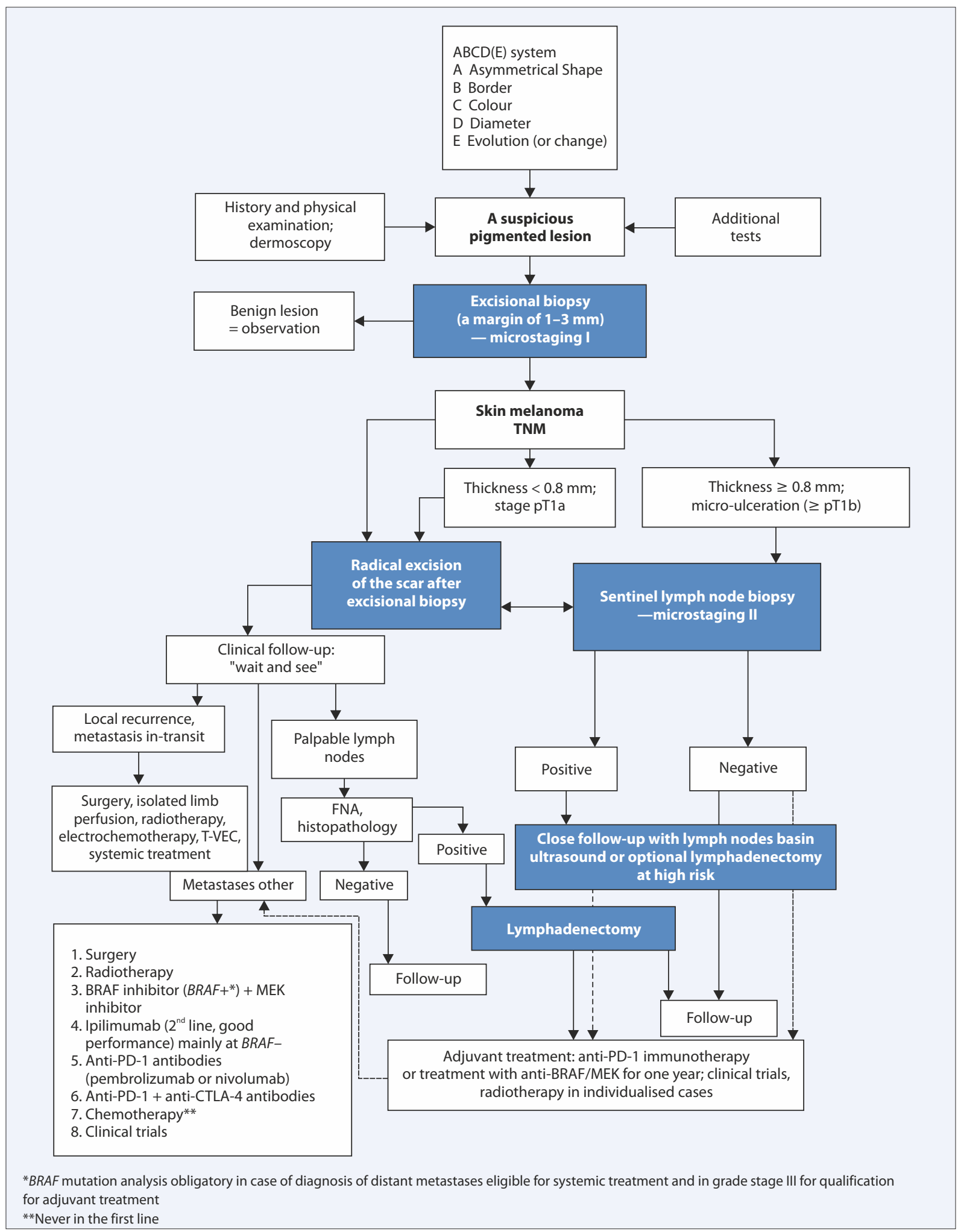

Figure 2. A schedule of diagnostic and therapeutic recommendations in patients with skin melanoma. FNA — fine-needle aspiration biopsy; TNM (tumour-node-metastasis) — classification of tumour/node/metastasis stage

the basis of examination of the material collected by fine-needle biopsy (in special cases — surgical biopsy) from enlarged and clinically suspected lymph nodes or in some cases in the confirmation of the presence of metastasis in sentinel nodes unsuspected clinically (microstaging II) [1, 10, 18]. 
Table 4. Summary of the recommendations of the National Comprehensive Cancer Network (NCCN) v. 1.2020, European Organisation for Research and Treatment of Cancer (EORTC), and the European Society of Medical Oncology (ESMO) concerning the final margin of the radical excision of the primary melanoma site depending on the Breslow thickness

\begin{tabular}{lc}
$\begin{array}{l}\text { Melanoma thickness } \\
\text { (Breslow) }\end{array}$ & $\begin{array}{c}\text { Recommended clinical } \\
\text { margin }\end{array}$ \\
\hline In situ & $0.5 \mathrm{~cm}$ \\
\hline$\leq 2.0 \mathrm{~mm}$ & $1 \mathrm{~cm}$ \\
\hline$>2.0 \mathrm{~mm}$ & $2 \mathrm{~cm}$ \\
\hline
\end{tabular}

\section{Therapeutic lymphadenectomy}

Qualification of patients for lymphadenectomy should be based on a clinical exam, laboratory test (including LDH serum level), and imaging techniques. If the metastases to distant organs are suspected, a patient should have computed tomography or PET-CT (especially of the pelvis when metastases to the iliac and obturator lymph nodes are suspected) and MRI. Imaging exam of the central nervous system should be performed in the case of occurrence of clinical symptoms and in stage IIIC.

The extent of the therapeutic lymphadenectomy in skin melanoma is as follows (III, C):

- in the axilla all lymph nodes should be removed according to the anatomic definition (three groups of lymph nodes and the surrounding fascia: lower compartment - pectoral [anterior] and subscapular [lateral] lymph nodes, central compartment - central axillary lymph nodes, upper compartment - infraclavicular [deltopectoral] and apical lymph nodes);

- in the groin we should remove the lymph nodes of the inguinal-femoral lymph nodes located below the inguinal ligament in the femoral triangle together with the femoral fascia, iliac lymph nodes placed along the external iliac vessels (optionally also internal and common), as well as the lymph nodes of the obturator fossa (in the case of metastases diagnosed in the sentinel nodes the lymphadenectomy should be restricted to inguinal lymph nodes);

- in the cervical lymphatic confluence modified procedures may be applied. These procedures must be maximally radical. Usually the neck structures that contain superficial lymph nodes (anterior and posterior) and profound are dissected in one piece, limited posteriorly by profound jugular facia and frontally by the platysma muscle.

Sometimes it is necessary to perform lymphadenectomy in the popliteal or ulnar fossa.

\section{Local reoccurrence and in-transit metastases}

Terms: satellitosis (micro- or macroscopic), local reoccurrence, and in-transit metastases form a kind of continuity and represent different forms of one pathologic phenomenon. Usually a local reoccurrence (often even after a very wide excision of the primary site) represents spreading of melanomas through the regional lymphatic vessels (microsatellites become macrosatellites), which may then transform into in-transit metastases. That is why in the majority of elaborates the above-mentioned forms of relapse of melanoma are analysed together and have similar prognosis (10-year survival about $20-30 \%$ ). Surgery is an essential method to treat a local relapse and in-transit metastases. Therapy should be individualised and should consider the number metastases, their size, localisation, and clinical course (III, B). In the case of in-transit metastases surgical therapy includes excision of the countable changes $(<10)$ with a microscopic melanoma infiltration-free margin (it may be macroscopically narrow). In the case of a single relapse lesion another sentinel lymph node biopsy may be considered. In the case of in-transit dissemination of melanoma limb amputation is not recommended. In the case of multiple/non-resectable lesions one of the local therapeutic methods should be considered (ablation, radiotherapy, cryotherapy), intratumoural immunotherapy (talimogene laherparepvec - T-VEC, PV-10 or interleukin-22 - is not encompassed by the National Drug Reimbursement Program) or local immunotherapy (imiquimod is not registered for this indication) and electrochemotherapy (III, B) or systemic therapy. In the case of extensive, multiple lesions located on the limb an hyperthermic isolated limb perfusion chemotherapy is a method of choice (HILP), mostly with use of melphalan. This method may be used only by experienced and trained centres (individual decisions on refunding). If HILP is contraindicated, systemic therapy should be administered [1, 5, 8, 18-20].

\section{Adjuvant therapy}

Currently, dabrafenib with trametinib (only patients with mutations in the $B R A F$ gene), pembrolizumab and nivolumab (the latter also after grade IV metastasectomy) are registered for systemic (one year) adjuvant treatment in clinical practice in patients after radical primary surgery and lymphadenectomy, and complementary radiotherapy may only be considered in individual cases. The results of some recently published clinical studies indicate an improvement of survival rates after both adjuvant immunotherapy with use of immune checkpoint inhibitors and combined therapy with BRAF and MEK inhibitors (only patients with mutations in the $B R A F$ gene) (I, B). 
High doses of interferon $\alpha$-2b (INF $\alpha-2 \mathrm{~b}$ ) have been registered based on the positive result of one of three clinical studies by the Easter Cooperative Oncology Group (ECOG) - ECOG 1684 - in the United States of America and in the European Community - to treat patients with melanoma in clinical stage IIB-III. Low-dose INF $\alpha$-2b has been registered in Europe for patients with clinical stage II melanoma [21, 22]. The registration was based on the significant prolongation of the overall survival during a seven-year observation time. These results have not been proven during a longer observation time (12 years). The results of the studies showed a repeatable (10 from 17 studies) improvement in the disease-free survival rates. The recent meta-analysis showed a significant decrease by $17-18 \%$ of the relative disease relapse risk after the administration of the adjuvant therapy with use of INF $\alpha-2 \mathrm{~b}$. The clinical evidence concerning overall survival rates is weaker and is based mostly on the results of meta-analyses. The overall five-year survival benefit for the whole group of patients reaches about $3-5 \%$. The use of adjuvant therapy with INF $\alpha-2 b$ in patients with intermediate and high relapse risk melanomas should be individualised due to its controversial clinical value and toxicity (II, B). The results of meta-analyses show that an adjuvant therapy with INF $\alpha$-2b may be beneficial in patients with ulcerated primary melanoma lesion, especially with coexistent micrometastases (to the sentinel node but with absence of metastases to the clinically enlarged lymph nodes) (I, B) [23, 24]. Interferon is not refunded in Poland in adjuvant treatment and is less effective than other drugs currently used in adjuvant treatment.

Ipilimumab is registered in the United Stated for adjuvant therapy of patients after lymphadenectomy of involved regional lymph nodes. Randomised clinical trials [25] showed a significant improvement of disease-free survival and overall survival but with high toxicity of ipilimumab therapy (II, B) [26]. Ipilimumab is not registered for adjuvant treatment in Poland.

Nivolumab in a randomised study in patients after stage IIIB, IIIC, and IV metastases showed a $10 \%$ improvement in recurrence-free survival compared to ipilimumab with lower toxicity (I, A), which is now a registered indication [25]. Updated data with a longer follow-up period confirm the beneficial effect of nivolumab in adjuvant treatment for a year regardless of the PD-L1 expression status and $B R A F$ mutation with respect to RFS (HR 0.66) and DMFS (HR 0.76) [27], the percentage of 3-year progression free survivals was $58 \%$ and was over $10 \%$ better than for ipilimumab. Dabrafenib treatment with trametinib in patients with high-risk grade III $B R A F$ (grade IIIA $>1 \mathrm{~mm}$, IIIB/C) showed an improvement in recurrence-free survival and overall survival compared to placebo (I, A) [28, 29]. Actualized data from 4 -year observations confirm the advantage of treatment with dabrafenib together with trametinib (RFS: 54\%; HR: 0.49; DFS: 67\%; HR: $0.53)$ [29]. Moreover, a model was presented evaluating the cure rate after using adjuvant therapy, which was in this case $17 \%$. The results of the Keynote-054/EORTC 1325 study in 1019 patients also indicate a reduction in the risk of recurrence (HR for RFS 0.57) and DMFS using pembrolizumab adjuvant treatment for one year, compared to placebo, in patients with grade III resection risk (IIIA with micrometers $>1 \mathrm{~mm}$, IIIB and IIIC) (I, B) [30]. This indicates the need for an absolute multidisciplinary evaluation of all patients with melanoma in stage II-IV. After a positive sentinel node biopsy, only adjuvant systemic treatment can be used without adjuvant lymphadenectomy. These drugs are available in Poland at present only in the scope of the Rescue Access to Drug Technology (RDTL) and have obtained a favorable opinion of AOTMiT for this indication.

Other methods of immunotherapy (e.g. interleukin-2), vaccines, or cytotoxic drugs have no clinical value in the adjuvant, postoperative therapy of melanomas.

In some individual cases, after surgical therapy of high-risk melanomas, an adjuvant radiotherapy (RT) may be applied. A dosing schedule includes - depending on the localisation of the melanoma lesion - hypofractionation, 3-8 Gy per fraction, or conventional fractioning. Indications for adjuvant radiotherapy after the primary tumour excision include: diagnosis of desmoplastic melanoma excited with narrow margins, presence of 'positive' surgical margins (especially after excision of the local reoccurrence), presence of satellite foci, significant neurotropism. In the case of excision of local reoccurrence and lymphadenectomy due to metastases to the regional lymph nodes, indications for adjuvant RT are: presence of extracapsular node infiltrations, involvement of $\geq 4$ lymph nodes (clinical stage IIIC), diameter of a metastasis $>3 \mathrm{~cm}$, detection of metastases to cervical lymph nodes (from two metastatic lymph nodes or when a metastasis measures at least $2 \mathrm{~cm}$ ), and reoccurrence after prior resection $[31,32]$. The results of the only completed randomised clinical trial assessing the value of adjuvant RT (48 Gy in 20 fractions) after lymphadenectomy in the case of high-relapse-risk melanoma confirmed the improvement of local control in patients receiving radiation. $\mathrm{RT}$ had no impact on overall survival rate and resulted in a higher ratio of locoregional complications and deterioration of patients' quality of life. These results suggest that use of adjuvant RT should be limited (II, C) [33]. No adjuvant RT should be applied after CLND. 


\section{Exclusively radiotherapy}

Exclusively radiotherapy as a non-palliative treatment can be used only in the case of an extensive LMM tumour.

Palliative radiotherapy can be used for individual indications in the case of primary or metastatic tumours not responding to systemic treatment, non-resectable, painful or bleeding.

\section{Therapy of patients with advanced disease}

The results of treatment of skin melanomas in clinical stage IV are still unsatisfactory. The median overall survival time exceeds 12 months (and is significantly higher for new therapies), but about $20-30 \%$ of patient survive for five years.

The significant prognostic factors in patients with melanoma in clinical stage IV are: performance status (according to the ECOG scale), LDH activity, and localisation of the metastatic lesions. In the case of qualification of a patient with clinical stage IV melanoma for surgery or systemic therapy, disease should be staged by imaging exams or PET-CT (only in the case of isolated metastatic foci qualified for resection) [1].

In the case of secondary changes to the skin, soft tissues, and non-regional lymph nodes (M1a, better prognosis), it is always recommended to consider excision. A similar approach should be applied for isolated (not necessarily single) metastases to the visceral organs. In the case of unresectable lesions, the choice of therapeutic approach depends on the presence of metastases to the central nervous system (CNS). If the metastases to the CNS are present neurosurgical treatment and/or radiotherapy of the central nervous system (usually stereotactic or radiosurgery [34]) should be considered as a first-line therapy (the decision depends on the location and number of lesions) in order to delay the occurrence of bleeding or neurological disorders. Radiotherapy of the central nervous system may be a part of combined therapy during immunotherapy (preferred) and during BRAF protein molecularly targeted therapy (II, B). There are no indications for irradiation of the whole brain (WBRT) in the scope of adjuvant treatment after local treatment of melanoma metastases to the CNS, as this does not improve treatment ourcomes. Detailed indications on treating melanoma metastases to the brain have been published [35]. RT is also used in palliative therapy in patients with metastases to soft tissues (ulceration, pain) and to bones (pain).

The advance in therapy of advanced melanoma, considering the low efficacy of cytotoxic agents, results from the use of nonspecific immunotherapy with use of monoclonal antibodies anti-CTLA4 (ipilimumab) or anti-PD1 (nivolumab, pembrolizumab), which inhibit the systemic mechanisms of immunosuppression in order to induce an antineoplastic response (activation of lymphocytes T) as well as from the use of molecularly targeted therapies with use of serine-threonine kinases inhibitors (dabrafenib with trametinib, vemurafenib with cobimetinib or encorafenib with binimetinib, where the last drug combination is not refunded) (I, A). Systemic treatment should be provided in centers having a full range of therapeutic possibilities [36]. Patients with advanced melanoma should still be referred and screened for prospective clinical trials.

Dacarbazine is the only registered cytotoxic drug for advanced melanoma. Its efficacy is limited (objective response rate $-15 \%$ of patients, median duration of response four months) [1]. The only registered scheme of dacarbazine therapy is a five-consecutive-day schedule with a daily dose of $200 \mathrm{mg} / \mathrm{m}^{2}$; an alternative schedule of administering a higher dose of a drug (850-1000 mg/m² every three weeks) has not formally been accepted; however, this alternative is considered useful in clinical practice. Paclitaxel in monotherapy or in combination with dacarbazine does not improve the duration of the response to the second-line therapy. Randomised trials in patients did not confirm higher efficacy of a polychemotherapy schedule including dacarbazine combined with cisplatin, vinca alkaloids (e.g. vinblastine) and nitrosamine derivates (e.g. carmustine) and tamoxifen. Use of biochemotherapy (chemotherapy combined with interleukin-2 and INF $\alpha$-2b) does not improve melanoma patients' overall survival rates compared to chemotherapy. The results of clinical studies indicate that interleukin-2 in monotherapy or combined with IFN $\alpha$-2b slightly improves the overall response rate, with no influence on the overall survival rate. The toxicity of this therapy is significant. Currently the use of chemotherapy should be limited to lifesaving situations after failure of the molecularly targeted therapies or immunotherapy (I, A).

\section{Immunotherapy}

Ipilimumab has been registered in the therapy of patients with advanced melanomas and resulted in significantly higher overall survival rates (a difference of about 3.5 months) compared to peptide vaccine gp100 in a second-line therapy, with no impact on the disease-free progression time $[37,38]$. Kinetics and time of response duration on ipilimumab therapy are different than for classical chemotherapy. The benefit of therapy is observed only after 3-4 months of therapy, which limits its application to patients with advanced melanoma with minimal symptoms, good performance status, and low disease course as well as (considering the safety profile) to patients with no autoimmune diseases. Due to late objective response occurrence, a reliable evaluation of the efficacy of ipilimumab therapy should be done after 12 weeks of treatment. Moreover, in the early phase of 
the therapy a phenomenon of paradoxical progression (so-called pseudo progression) due to infiltration of the tumours by the immunocompetent cells may occur. The immunological response criteria should be applied in order to get objective imaging examination evaluation of the ipilimumab efficacy [37-39]. Currently there are no known predictive factors of response to ipilimumab. A recommended dosing schedule is $3 \mathrm{mg} / \mathrm{kg}$ of body weight, administered every three weeks, up to four doses (I, A).

The objective overall response rate to ipilimumab therapy is low (about 10\%), and long-term benefits are observed in a limited number of patients (20-25\%); however, they are characterised by long-lasting responses (the longest observation reaches 10 years). Adverse events related to autoimmunological reactions constitute a major problem of ipilimumab therapy (grade 3-4 adverse events occur in about 20-25\% of patients). The most common immunological adverse events include: skin changes, colitis (diarrhoea), hepatotoxicity, and endocrinopathies (including insufficiency of pituitary and thyroid gland). Occurrence of these syndromes in a patient treated with ipilimumab should result in an urgent referral of this patient to a medical centre experienced in treating complications of immunotherapy. In the case of intensified symptoms that disenable transportation, corticosteroids should be immediately administered (prednisolone [or equivalent] 1-2 mg/kg of body weight), and further therapy should be applied in collaboration with, or with assistance of, a referral centre. The appropriate algorithms of proceeding are accessible [38] and should be rigorously implemented from the moment of the occurrence of first symptoms suggesting immunological toxicity.

Ipilimumab therapy should be applied only in tertiary referral centres that provide holistic diagnostic and therapeutic proceedings. It is not recommended that this therapy be started in inexperienced centres with limited therapeutic options.

Currently, immunotherapy in skin melanomas is mostly related to the usage of immune control checkpoint PD-1 in monotherapy (nivolumab in fixed does $240 \mathrm{mg}$ every two weeks or $480 \mathrm{mg}$ every four weeks or pembrolizumab $200 \mathrm{mg}$ every three weeks or $400 \mathrm{mg}$ every six weeks) (I, A) [40-42] or in combination with anti-CTLA-4 antibodies (I, B) [43]. These agents have been proven in clinical practice, in monotherapy or in combination with ipilimumab, to give long-lasting clinical benefit in some patient with advanced melanomas and significant response rates (reaching 50\%) and one-year survival rates of $70-80 \%$. The use of nivolumab or pembrolizumab results in two-year survival rates of 50-60\% (median survival exceeds two years; three-year survival rate reaches about $45 \%$ ), with acceptable toxicity (about $15 \%$ in grade $3 / 4$, which is significantly less than for ipilimumab); however, the most severe symptom also results from autoimmune toxicity. Clinical studies confirmed a higher efficacy of pembrolizumab concerning the overall survival and disease-free survival time compared to ipilimumab in first-line therapy and compared to chemotherapy after failure of prior therapy [40-42]. In recently published results of a clinical trial that compared efficacy of nivolumab in monotherapy, ipilimumab in monotherapy, and a combination of both drugs, nivolumab was revealed to be more effective than ipilimumab (the median disease-free survival time reached, respectively, 6.9 vs. 2.9 months); however, the combination of both drugs had the highest (in comparison with ipilimumab) efficacy (the median disease-free survival was 11.5 months) [43]. The results of combined ipilimumab and nivolumab therapy were better when a $B R A F$ gene mutation was present; however, in the whole group of patients [44] and after 5 year overall survivals in the combined branch were $52 \%$ (thus the median exceeded 60 months) in comparison with $44 \%$ for nivolumab monotherapy [45]. The adverse events in Common Terminology Criteria for Adverse Events (CTCAE) grade 3-4 were significantly more frequent in the combined therapy arm $(56.5 \%)$ compared to $19 \%$ in the nivolumab and $27 \%$ in the ipilimumab arm. Combined immunotherapy and not anti-PD1 monotherapy can be the preferred option in patients with a very good performance status with poorer prognostic factors (including the $B R A F$ mutation, with high $\mathrm{LDH}$ levels and asymptomatic metastases to the brain) (II, B) $[46,47]$. Nivolumab with ipilimumab at present is not refunded in Poland.

In the clinical study a therapy with anti-PD-L1 antibody, pembrolizumab, was maximally continued for two years. In the group of 104 patients who accomplished the two-year therapy period, 102 persons (98\%) are still alive while the nine-month disease progression-free survival rate reached $91 \%$ (which means that in the majority of patients disease control was maintained even when the active therapy had been stopped). Based on available literature data, it is now possible to consider discontinuing immunotherapy with anti-PD1 antibodies in patients who have an objective response after at most two years of treatment (CR, PR)/clinical benefit (II, B) [48].

In light of the presented results of the clinical studies, ipilimumab should not constitute an essential type of immunotherapy in patients with advanced melanomas, because it is less efficient than anti PD-L1 antibodies and has a worse safety profile. The therapy should be started from anti PD-L1 (nivolumab or pembrolizumab) in monotherapy (I, A). The issue of combined therapy with anti-CTLA-4 antibodies requires further investigation, the use of combination of anti-CTLA- 4 with ant-PD- 1 is specifically justified in patients with asymptomatic brain metastases to CNS (II, B). 


\section{Molecularly targeted therapy}

The presence ofmutation of the RAS/RAF/MEK/ERK MAP kinase pathway is detected in $75 \%$ of skin cancers. The major mechanism leading to hyperactivity of RAS/RAF/MAPK pathway I skin melanoma is a mutation of a kinase BRAF encoding gene mutation. Somatic mutations in $B R A F$ gene are observed in $50-70 \%$ of skin cancers occurring on skin areas not exposed to long-term solar radiation. Published in the year 2011, the results of the registration phase III study of vemurafenib use in first-line therapy in patients with present $B R A F$ $V 600$ mutation showed $48 \%$ overall response to therapy in patients receiving BRAF inhibitor (BRAFi) compared to $5 \%$ in patients on dacarbazine, as well as significant improvement of disease progression time (five months difference) and of overall survival (three months difference) [49]. Vemurafenib has been registered to treat patients with advanced melanoma with presence of $B R A F$ mutation (testing for this mutation is possible in Polish centres with use of a validated test) (I, A). Even though in the majority of patients, resistance to therapy will develop (median disease progression-free survival totals $6-7$ months), the results of phase II-III revealed a 13-16-month-long median overall survival time, in patients with metastatic melanoma, which is significantly better than any other reported survival benefit in this subset of patients. Vemurafenib is characterised by significant skin toxicity (hypersensitivity to UV radiation), hepatotoxicity typical for kinase inhibitors, and by formation of secondary neoplasms (cancer or keratoacanthoma of the skin in about $20 \%$ of treated patients). The secondary skin neoplasms may develop within a few weeks after the onset of the therapy with vemurafenib. Diagnosis of secondary skin cancers requires local therapy but not interrupting the drug. The adverse events quite often require reduction of vemurafenib dose. In the year 2012 a therapeutic efficacy of another BRAF inhibitor, dabrafenib, was proven (characterised by efficacy similar to vemurafenib but by a different toxicity profile, e.g. lower skin toxicity). The median disease progression-free time reached 6.7 months for dabrafenib vs. 2.9 months for dacarbazine, whereas the median overall survival time on dabrafenib therapy reported in the year 2013 reached 18.2 months (I, A) [50]. In a phase III trial, the efficacy of MEK inhibitor (MEKi) - trametinib has also been confirmed in patients with metastatic melanomas harbouring $B R A F$ gene mutation (I, B) [51]. The efficacy of MEK inhibitors has also been observed in patients with $N R A S$ gene mutation [52]. The results of recent studies (COMBI-d, COMBI-v, coBRIM and COLUMBUS) showed that in patients with metastatic melanomas with $B R A F$ gene mutation the use of a combination of BRAF and MEK inhibitors (dabrafenib and trametinib or vemurafenib with cobimetinib or encorafenib with binimetinib) yields better results than monotherapy and no increase of toxicity (I, A) [53-59]. The median overall survival time on the combination of both drugs was improved to about 23-33 months and a median disease progression time of 12-14 months. The best overall survival is achieved in patients with normal $\mathrm{LDH}$ activity and serum concentration and less than three organs involved with metastases. The first two combinations are currently accessible in Poland in the Drug Program in the first- or second-line therapy in patients with advanced melanoma with confirmed presence of $B R A F$ V600 mutation.

The above-mentioned drugs have a beneficial influence also in patients with stable and/or asymptomatic metastases to the brain, and until now this localisation was inaccessible for the systemic therapy of melanoma. Patients with melanoma and $B R A F$ gene mutation, in whom asymptomatic brain metastases have been detected, may receive a first-line therapy with BRAF inhibitor (in combination with MEK inhibitor).

A new option of the molecularly targeted therapy is to restart the combined therapy with BRAF and MEK inhibitors after this therapy has been stopped due to disease progression. A phase II study revealed that restarting therapy with dabrafenib and trametinib resulted in partial remission in eight of 25 patients $(32 \%)$ and in stabilisation of the disease in another $40 \%$ of patients. The median disease progression-free time to so-called 'rechallenge' reached 4.9 months [60]. The analysis of data of 116 patients with advanced melanoma, who had received therapy with BRAF inhibitor, progressed, and received another therapeutic modality, and then were restarted on combined therapy with $\mathrm{BRAF} \pm \mathrm{MEK}$ inhibitor, was presented at the ASCO meeting in 2017. The median time of treatment duration was 9.4 and 7.7 months for the primary and reused molecularly targeted therapy, respectively. After restarting the use of BRAF \pm MEK inhibitors the response rate was $43 \%$ : complete response rate $3 \%$, partial response rate $39 \%$, stabilisation of the disease $24 \%$, and progression of the disease $30 \%$ (no data $4 \%$ ). The median overall survival time form the restart of the therapy reached 9.8 months (II, B) [61, 62].

BRAF inhibitors ( + MEK inhibitors) induce a prompt response and neoplasm control in the majority of patients with advanced melanomas with present $B R A F$ gene mutation. However, the response duration is limited due to activation of mechanisms of resistance to therapy. Due to these characteristics this therapy should be considered as a treatment of choice in patients with symptomatic disease and/or high tumour mass. There are no final data concerning the optimal sequence of immunotherapy and molecularly targeted therapy in patients with melanomas with presence of $B R A F$ gene mutation. However, the activity of BRAF inhibitor is 


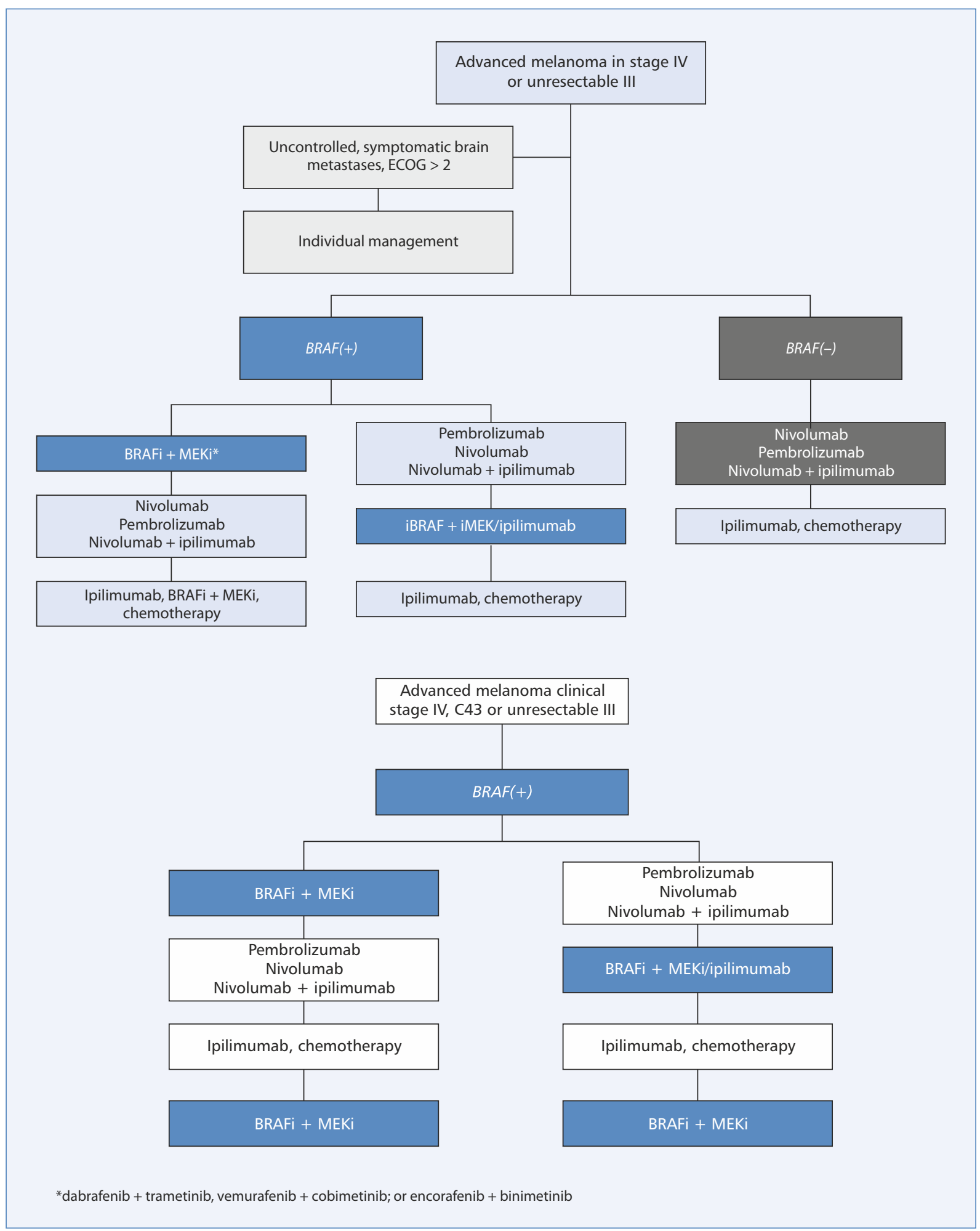

Figure 3. General approach to systemic treatment in patients with advanced stage IV or unresectable III melanomas and detailed plan of systemic treatment in patients with advanced stage IV or unresectable III melanomas with BRAF mutation, showing the possibility of restarting the treatment with BRAF + MEK inhibitors (rechallenge). BRAFi - BRAF inhibitor; MEKi — MEK inhibitor

maintained after immunotherapy and of immunotherapy (anti-PD-L1) after treatment with BRAF inhibitors (Fig. 3) [63]. In rare cases of patients with melanomas carry- ing some KIT gene mutations, the activity of KIT kinase inhibitors has been observed which are not refunded for this indication (II, B) [64]. 
Table 5. Exams recommended in monitoring melanoma patients

\begin{tabular}{|c|c|}
\hline $\begin{array}{l}\text { Clinical stage of } \\
\text { melanoma }\end{array}$ & Type of exam \\
\hline $\begin{array}{l}\text { Early melanomas after the } \\
\text { excision of the primary site } \\
\text { without any metastases to } \\
\text { the lymph nodes (clinical } \\
\text { stages IA-IB) }\end{array}$ & $\begin{array}{l}\text { Physical examination and anamnesis, especially a careful examination of } \\
\text { the whole skin surface and of the regional lymph nodes as well as of the } \\
\text { area of the scare post excision of melanoma } \\
\text { Radiologic image (RT) of the chest - optionally } \\
\text { Other exams (e.g. US, CT) in the case of presence of suspected symptoms } \\
\text { Ultrasound of regional nodes when no sentinel node biopsy has been } \\
\text { performed, in skin melanomas } \geq \mathrm{pT} 1 \mathrm{~b} \\
\text { There are no indications for any additional test except for physical exam } \\
\text { in patients post excision of melanoma pT1a } \\
\text { Patients should be trained to perform a self-control examination }\end{array}$ \\
\hline
\end{tabular}

\section{Frequency \\ of control exams}

Every 6-12 months during

the first 5 years, then

once a year (follow-up may be done outside the

specialist centre)
Locally advanced melanomas post excision

of the primary site without metastases to regional lymph nodes (clinical stages IIA-IIC)
Physical examination and anamnesis, especially a careful examination of a whole skin surface and of the regional lymph nodes as well as of the area of the scare post excision of melanoma

Radiologic image (RT) of the chest, ultrasound of the abdomen Other tests (e.g. CT) in the case of presence of suspected symptoms Ultrasound of regional nodes when no sentinel node biopsy has been performed, in skin melanomas $\geq \mathrm{pT} 1 \mathrm{~b}$

In patients with clinical stage IIB-IIC a CT exam may be done every 6-12 months and optionally MRI of CNS once a year (during the first 2-3 years)

Patients should be trained to perform a self-control examination. In clinical stage IIC more intensive monitoring schedules may be used as in clinical stage III

Post excision of the Physical examination and anamnesis. Especially a careful examination of metastases to the regional lymph nodes or of a local relapse/satellite or in-transit lesion (clinical stages IIIA-IIID) or observation after detection of metastasis to the sentinel lymph node without complementary lymphadenectomy

a whole skin surface and of the regional lymph nodes as well as of the area of the scare post excision of melanoma

Radiologic image (RT) of the chest

Blood morphology and biochemistry (liver tests and activity of lactate dehydrogenase) - optionally

Ultrasound examination of lymphatic drainage every 4-6 months in case of finding a positive sentinel node without performing lymphadenectomy Ultrasound of abdomen and eventually of the regions of the removed lymph nodes

CT exam of the chest, abdomen, and pelvis every 6-12 months and optionally in clinical stage IIIC/IIID, once a year a MRI of the brain (during the first 3 years)

Patients should be trained to perform a self-control examination

\begin{tabular}{lll}
\hline After therapy of distant & Evaluation of the imaging exams depending on the localisation of the & An individual monitoring \\
metastases (clinical stage IV) & $\begin{array}{l}\text { measurable metastatic sites } \\
\text { Serum activity of LDH }\end{array}$ & schedule for each patient
\end{tabular}

US — ultrasonography; CT — computed tomography; MR — magnetic resonance; LDH — lactate dehydrogenase

\section{Follow-up after therapy completion}

The frequency and type of control examinations as well as duration of the observation should be established based on the individual disease relapse risk (which depends on the initial clinical stage of the disease). However, we should bear in mind that the relapse may occur even 10 years after the primary treatment $[65,66]$ (Table 5). The relapse risk is the highest in the first three years post therapy. That is why it is recommended that a more intense schedule of control exams should be applied in this period in order to detect a loco-regional relapse, which may be cured by surgery. Assessment of scars post primary site excision and post lymphadenectomy constitutes the most important part of the observation. The evaluation of the regional lymph confluence should be done carefully (a possible in-transit dissemination). To evaluate the local lymph nodes, we can use palpation and ultrasonography. A patient may detect a majority of loco-regional relapses, and that is why he/she should be trained to make a self-control of the area of the melanoma excision and of the regional lymph nodes. There 
are some premises that a less intensive control schedule has no negative impact on the survival in patients with early melanomas.

Imaging exams are not recommended in asymptomatic patients with clinical stage IA-IIA. Imaging exams (e.g. CT exam) may be considered in asymptomatic patients with clinical stage IIB-IIIC during the first 2-3 years of follow-up (taking into consideration the availability of some new, effective drugs in the therapy of disseminated melanomas (IV, B). The earlier data evaluating the intensive schedule of the control imaging exams demonstrated only a minimal benefit - maximally two months prolongation of the overall survival time). Then, in patients with clinical symptoms suggesting the presence of distant metastases (liver enzymes elevation, bone pains, neurological symptoms, cough, and weakness) detailed imaging diagnostics should be done, with CT, MRI, PET-CT, and bone scintigraphy included.

During the control exams we should carefully check not only the area of the primary melanoma lesion but also the whole skin surface. Melanoma patients have a statistically higher risk of developing a lesion of melanoma or of another skin cancer.

Additional information for patients can be found on the web sites for example of scientific societies (e.g. www.akademiaczerniaka.pl). The scheme of control check-ups should be given to the patient in writing.

\section{Summary}

Excisional biopsy of the suspected pigmented moles, which may be early melanomas, is essential to diagnose and assess the main risk factors of melanoma (microstaging I). Early diagnosis and removal of melanoma not only improves the prognosis but also gives a chance of cure in nearly $90 \%$ of patients. Usually the pigmented changes with transversal axis dimensions not exceeding $2 \mathrm{~cm}$ may be removed in an outpatient clinic during an excisional biopsy. The next stages of the proceedings include qualification of a patient to a radical, wide scar excision with appropriate surgical margins $0.5-2 \mathrm{~cm}$ depending on the thickness according to Breslow) and performing a sentinel node biopsy (stage $\geq$ pT1b). In the case of clinical metastases to the regional lymph nodes a radical lymphadenectomy is a method of choice. It is recommended that patients with high-risk melanoma be qualification in systemic adjuvant treatment (nivolumab, pemrbolizumab, dabrafenib with trametinib). A schedule of diagnostic and therapeutic recommendations in patients with skin melanoma is shown in Figure 3.

The presence of distant metastases is still associated with poor prognosis. It is recommended that patients with generalised disease be treated in clinical trials. $B R A F$ mutation should be tested in all patients with advanced disease or with high disease relapse risk (III). Long-term survival is seen mostly in patients in clinical stage IV, who have had resection of singular metastatic lesions. In patients with present $B R A F V 600$ gene mutation, mostly in first-line therapy, a BRAF inhibitor may be used (in combination with MEK inhibitor). Immunotherapy with anti PD-1 antibodies (nivolumab or pembrolizumab) or alternatively ipilimumab (anti-CTLA-4 antibody in monotherapy or in combination with anti-PD-1) may be used independently of the $B R A F$ mutation presence. The optimal sequence of therapy (especially in the case of $B R A F$ mutation) has not been assessed. The use of combined therapy with BRAF and MEK inhibitors involves a high response rate (about 70\%) and rapid alleviation of symptoms of the disease. Therapy with anti-PD-1 antibodies results in lower response rates, but in the majority of patients the response is durable. Supplementary radiotherapy may be considered in cases at high recurrence risk after local surgery especially in the case of limited indications for systemic treatment.

\section{References}

1. Rutkowski P. Złośliwe nowotwory skóry. Via Medica, Gdańsk 2014

2. Didkowska J, Wojciechowska U, Olasek P. Nowotwory złośliwe w Polsce w 2017 roku. Cancer in Poland in 2017. Warszawa 2019

3. Wojciechowska U, Didkowska J. Zachorowania i zgony na nowotwory złośliwe w Polsce. Krajowy Rejestr Nowotworów, Narodowy Instytut Onkologii im. Marii Skłodowskiej-Curie - Państwowy Instytut Badawczy. Dostępne na stronie http://onkologia.org.pl/raporty/ dostęp z dnia 05/04/2020

4. Wouters MW, Michielin O, Bastiaannet E, et al. ECCO essential requirements for quality cancer care: Melanoma. Crit Rev Oncol Hematol. 2018; 122: 164-178, doi: 10.1016/j.critrevonc.2017.12.020, indexed in Pubmed: 29458785

5. Michielin O, Akkooi Av, Ascierto PA, et al. Cutaneous melanoma: ESMO Clinical Practice Guidelines for diagnosis, treatment and follow-up. Annals of Oncology. 2019; 30(12): 1884-1901, doi: 10.1093/annonc/mdz411.

6. Gajda M, Kaminska-Winciorek G. Do not let to be late: overview of reasons for melanoma delayed diagnosis. Asian Pac J Cancer Prev. 2014; 15(9): 3873-3877, doi: 10.7314/apjcp.2014.15.9.3873, indexed in Pubmed: 24935566

7. Kamińska-Winciorek G. Placek W. The most common mistakes on dermatoscopy of melanocytic lesions. Postepy Dermatol Alergol. 2015; 32(1): 33-39, doi: 10.5114/pdia.2014.44029, indexed in Pubmed: 25821425

8. NCCN Guidelines. Cutaneous melanoma version 1.2020

9. Elder E, Massi D, Scolyer RA, et al. Classification of Skin Tumours 4th Edition. International Agency for Research on Cancer 2018.

10. Morton DL. Thompson JF, Cochran AJ, et al. MSLT Group. Final trial report of sentinel-node biopsy versus nodal observation in melanoma. N Engl J Med. 2014; 370(7): 599-609, doi: 10.1056/NEJMoa1310460, indexed in Pubmed: 24521106.

11. Wong SL, Balch CM, Hurley P, et al. American Society of Clinical Oncology, Society of Surgical Oncology. Sentinel lymph node biopsy for melanoma: American Society of Clinical Oncology and Society of Surgical Oncology joint clinical practice guideline. J Clin Oncol. 2012; 30(23): 2912-2918, doi: 10.1200/JCO.2011.40.3519, indexed in Pubmed: 22778321.

12. Balch CM, Gershenwald JE, Soong SJ, et al. Final version of 2009 AJCC melanoma staging and classification. J Clin Oncol. 2009; 27(36): 6199-6206, doi: 10.1200/JCO.2009.23.4799, indexed in Pubmed: 19917835

13. Nowecki ZI, Rutkowski P, Michej W. The survival benefit to patients with positive sentinel node melanoma after completion lymph node dissection may be limited to the subgroup with a primary lesion Breslow 
thickness greater than 1.0 and less than or equal to $4 \mathrm{~mm}$ (pT2-pT3). Ann Surg Oncol. 2008; 15(8): 2223-2234, doi: 10.1245/s10434-008 9965-3, indexed in Pubmed: 18506535

14. Faries M, Thompson J, Cochran A, et al. Completion Dissection or Observation for Sentinel-Node Metastasis in Melanoma. New England Journal of Medicine. 2017; 376(23): 2211-2222, doi: 10.1056/nejmoa1613210.

15. Leiter U, Stadler R, Mauch C, et al. German Dermatologic Cooperative Oncology Group (DeCOG). Complete lymph node dissection versus no dissection in patients with sentinel lymph node biopsy positive melanoma (DeCOG-SLT): a multicentre, randomised, phase 3 trial. Lance Oncol. 2016; 17(6): 757-767, doi: 10.1016/S1470-2045(16)00141-8, indexed in Pubmed: 27161539.

16. van Akkooi ACJ, Nowecki Zl, Voit C, et al. Sentinel node tumor burden according to the Rotterdam criteria is the most important prognostic factor for survival in melanoma patients: a multicenter study in 388 patients with positive sentinel nodes. Ann Surg. 2008; 248(6): 949-955 doi: 10.1097/SLA.0b013e31818fefe0, indexed in Pubmed: 19092339.

17. Gershenwald JE, Scolyer RA, Hess KR. Melanoma of the skin. AJCC Cancer Staging Manual. Eight Edition. Springer 2017.

18. Testori A, Rutkowski P, Marsden J, et al. Surgery and radiotherapy in the treatment of cutaneous melanoma. Ann Oncol. 2009; 20 Suppl 6: vi22-vi29, doi: 10.1093/annonc/mdp257, indexed in Pubmed: 19617294

19. Mali $B$, Jarm T, Snoj $M$, et al Antitumor effectiveness of electrochemotherapy: a systematic review and meta-analysis. Eur J Surg Oncol. 2013; 39(1): 4-16, doi: 10.1016/j.ejso.2012.08.016, indexed in Pubmed: 22980492

20. Andtbacka RHI, Kaufman HL, Collichio F, et al. Talimogene Laherparepvec Improves Durable Response Rate in Patients With Advanced Melanoma J Clin Oncol. 2015: 33(25): 2780-2788, doi: 10.1200/JCO.2014.58.3377, indexed in Pubmed: 26014293.

21. Eggermont AMM, Gore M. Randomized adjuvant therapy trials in melanoma: surgical and systemic. Semin Oncol. 2007; 34(6) 509-515, doi: 10.1053/j.seminoncol.2007.09.003, indexed in Pubmed: 18083374

22. Sondak VK, Gonzalez RJ, Kudchadkar R. Adjuvant therapy for melanoma: a surgical perspective. Surg Oncol Clin N Am. 2011: 20(1): 105-114, doi: 10.1016/j.soc.2010.09.001, indexed in Pubmed: 21111961.

23. Eggermont AMM, Chiarion-Sileni V, Grob JJ, et al. Prolonged Survival in Stage III Melanoma with Ipilimumab Adjuvant Therapy. N Engl J Med. 2016; 375(19): 1845-1855, doi: 10.1056/NEJMoa1611299, indexed in Pubmed: 27717298.

24. Eggermont AMM, Suciu S, Testori A, et al. Ulceration and stage are predictive of interferon efficacy in melanoma: results of the phase III adjuvant trials EORTC 18952 and EORTC 18991. Eur J Cancer 2012; 48(2): 218-225, doi: 10.1016/j.ejca.2011.09.028, indexed in Pubmed: 22056637

25. Weber J, Mandala M, Del Vecchio M, et al. CheckMate 238 Collaborators. Adjuvant Nivolumab versus Ipilimumab in Resected Stage III or IV Melanoma. N Engl J Med. 2017; 377(19): 1824-1835, doi: 10.1056/NEJMoa1709030, indexed in Pubmed: 28891423

26. Coens C, Suciu S, Chiarion-Sileni V, et al. Adjuvant ipilimumab versus placebo after complete resection of high-risk stage III melanoma (EORTC 18071): a randomised, double-blind, phase 3 trial. Lancet Oncol. 2015; 16(5): 522-530, doi: 10.1016/S1470-2045(15)70122-1, indexed in Pubmed: 25840693.

27. Weber J, Mandalà M, Vecchio MD, et al. Adjuvant therapy with nivolumab (NIVO) versus ipilimumab (IPI) after complete resection of stage III/IV melanoma: Updated results from a phase III trial (CheckMate 238). Journal of Clinical Oncology. 2018; 36(15 suppl): 9502-9502 doi: 10.1200/jco.2018.36.15 suppl.9502.

28. Long GV, Hauschild A, Santinami M, et al. Adjuvant Dabrafenib plus Trametinib in Stage III BRAF-Mutated Melanoma. N Engl J Med. 2017; 377(19): 1813-1823, doi: 10.1056/NEJMoa1708539, indexed in Pubmed: 28891408.

29. Hauschild A, Dummer $R$, Schadendorf $D$, et al Longer Follow-Up Confirms Relapse-Free Survival Benefit With Adjuvant Dabrafenib Plus Trametinib in Patients With Resected V600-Mutant Stage III Melanoma. J Clin Oncol. 2018; 36(35): 3441-3449, doi: 10.1200/JCO.18.01219, indexed in Pubmed: 30343620.

30. Eggermont AMM, Blank CU, Mandala M, et al. Adjuvant Pembrolizumab versus Placebo in Resected Stage III Melanoma. N Engl J Med. 2018; 378(19): 1789-1801, doi: 10.1056/NEJMoa1802357, indexed in Pubmed: 29658430.

31. Burmeister $\mathrm{BH}$, Henderson MA, Ainslie J, et al. Adjuvant radiotherapy versus observation alone for patients at risk of lymph-node field relapse after therapeutic lymphadenectomy for melanoma: a randomised trial. Lancet Oncol. 2012; 13(6): 589-597, doi 10.1016/S1470-2045(12)70138-9, indexed in Pubmed: 22575589.
32. Ballo MT, Ang KK. Radiotherapy for cutaneous malignant melanoma: rationale and indications. Oncology (Williston Park). 2004; 18(1) 99-107; discussion 107, indexed in Pubmed: 14768409.

33. Henderson MA, Burmeister $\mathrm{BH}$, Ainslie J, et al. Adjuvant lymph-node field radiotherapy versus observation only in patients with melanoma at high risk of further lymph-node field relapse after lymphadenectomy (ANZMTG 01.02/TROG 02.01): 6-year follow-up of a phase 3, randomised controlled trial. Lancet Oncol. 2015; 16(9): 1049-1060, doi: 10.1016/S1470-2045(15)00187-4, indexed in Pubmed: 26206146.

34. Rutkowski P, Kiprian D, Dudzisz-Śledź M, et al. Management of brain metastases in melanoma. Oncol Clin Pract. 2018; 14: 148-155, doi 5603/ OCP.2018.0031

35. Rutkowski P, Kiprian D, Dudzisz-Śledź M, et al. Management of melanoma metastases in the brain. Nowotwory. Journal of Oncology. 2019; 69(3-4): 86-96, doi: 10.5603/njo.2019.0018.

36. Fogarty G, Dolven-Jacobsen K, Morton R, et al. Phase 3 internationa trial of adjuvant whole brain radiotherapy (WBRT) or observation (Obs) following local treatment of 1-3 melanoma brain metastases (MBMs) Journal of Clinical Oncology. 2019; 37(15 suppl): 9500-9500, doi: 10.1200/jco.2019.37.15 suppl.9500.

37. Hodi FS, O'Day SJ, McDermott DF, et al. Improved survival with ipilimumab in patients with metastatic melanoma. $\mathrm{N}$ Engl $\mathrm{J}$ Med. 2010; 363(8): 711-723, doi: 10.1056/NEJMoa1003466, indexed in Pubmed: 20525992

38. Świtaj T, Wysocki P, Wojtukiewicz M, et al. Ipilimumab - postęp w terapii chorych na zaawansowanego czerniaka. Onkol Prakt Klin. 2011; 7: 231-245.

39. Wolchok JD, Hoos A, O'Day S, et al. Guidelines for the evaluation of immune therapy activity in solid tumors: immune-related response criteria. Clin Cancer Res. 2009; 15(23): 7412-7420, doi: 10.1158/10780432.CCR-09-1624, indexed in Pubmed: 19934295.

40. Robert C, Long GV, Brady B, et al. Nivolumab in previously untreated melanoma without BRAF mutation. N Engl J Med. 2015; 372(4): 320-330, doi: 10.1056/NEJMoa1412082, indexed in Pubmed: 25399552.

41. Weber JS, D'Angelo SP, Minor D, et al. Nivolumab versus chemotherapy in patients with advanced melanoma who progressed after anti-CTLA-4 treatment (CheckMate 037): a randomised, controlled, open-label, phase 3 trial. Lancet Oncol. 2015; 16(4): 375-384, doi: 10.1016/S1470 2045(15)70076-8, indexed in Pubmed: 25795410.

42. Robert C, Schachter J, Long GV, et al. KEYNOTE-006 investigators, KEYNOTE-006 investigators. Pembrolizumab versus Ipilimumab in Advanced Melanoma. N Engl J Med. 2015; 372(26): 2521-2532, doi: 10.1056/NEJMoa1503093, indexed in Pubmed: 25891173

43. Larkin J, Chiarion-Sileni V, Gonzalez R, et al. Combined Nivolumab and Ipilimumab or Monotherapy in Untreated Melanoma. N Engl J Med. 2015; 373(1): 23-34

44. Wolchok J, Chiarion-Sileni V, Gonzalez R, et al. Overall Survival with Combined Nivolumab and Ipilimumab in Advanced Melanoma New England Journal of Medicine. 2017; 377(14): 1345-1356, doi: 10.1056/nejmoa1709684

45. Larkin J, Chiarion-Sileni V, Gonzalez R, et al. Five-Year Survival with Combined Nivolumab and Ipilimumab in Advanced Melanoma. N Eng J Med. 2019; 381(16): 1535-1546, doi: 10.1056/NEJMoa1910836, indexed in Pubmed: 31562797

46. Long G, Atkinson V, Lo S, et al. Combination nivolumab and ipilimumab or nivolumab alone in melanoma brain metastases: a multicentre randomised phase 2 study. The Lancet Oncology. 2018; 19(5): 672-681, doi: 10.1016/s1470-2045(18)30139-6.

47. Tawbi HA Forsyth PA, Algazi $\mathrm{A}$, et al. Combined Nivolumab and Ipilimumab in Melanoma Metastatic to the Brain. N Engl J Med. 2018; 379(8): 722-730, doi: 10.1056/NEJMoa1805453, indexed in Pubmed: 30134131

48. Robert C, Ribas A, Hamid O, et al. Durable Complete Response After Discontinuation of Pembrolizumab in Patients With Metastatic Melanoma. J Clin Oncol. 2018; 36(17): 1668-1674, doi 10.1200/JCO.2017.75.6270, indexed in Pubmed: 29283791

49. Chapman PB, Hauschild A, Robert C, et al. BRIM-3 Study Group. Improved survival with vemurafenib in melanoma with BRAF V600E mutation. N Engl J Med. 2011; 364(26): 2507-2516, doi: 10.1056/NEJMoa1103782, indexed in Pubmed: 21639808.

50. Hauschild A, Grob JJ, Demidov LV, et al. Dabrafenib in BRAF-mutated metastatic melanoma: a multicentre, open-label, phase 3 randomised controlled trial. Lancet. 2012; 380(9839): 358-365, doi: 10.1016/S0140-6736(12)60868-X, indexed in Pubmed: 22735384

51. Flaherty KT, Robert C, Hersey P, et al. METRIC Study Group. Improved survival with MEK inhibition in BRAF-mutated melanoma. N Engl J Med. 2012; 367(2): 107-114, doi: 10.1056/NEJMoa1203421, indexed in Pubmed: 22663011. 
52. Ascierto P, Berking $C$, Agarwala $S$, et al. Efficacy and safety of ora MEK162 in patients with locally advanced and unresectable or metastatic cutaneous melanoma harboring BRAFV600 or NRAS mutations. Journal of Clinical Oncology. 2012; 30(15 suppl): 8511-8511, doi: 10.1200/jco.2012.30.15 suppl.8511.

53. Robert C, Karaszewska B, Schachter J, et al. Improved overall survival in melanoma with combined dabrafenib and trametinib. N Eng J Med. 2015; 372(1): 30-39, doi: 10.1056/NEJMoa1412690, indexed in Pubmed: 25399551

54. Long GV, Stroyakovskiy D, Gogas H, et al. Dabrafenib and trametinib versus dabrafenib and placebo for Val600 BRAF-mutant melanoma a multicentre, double-blind, phase 3 randomised controlled trial. Lancet. 2015; 386(9992): 444-451, doi: 10.1016/S0140-6736(15)60898-4 indexed in Pubmed: 26037941.

55. Ascierto PA, McArthur GA, Dréno B, et al. Cobimetinib combined with vemurafenib in advanced BRAF(V600)-mutant melanoma (coBRIM) updated efficacy results from a randomised, double-blind, phase 3 trial. Lancet Oncol. 2016; 17(9): 1248-1260, doi: 10.1016/S1470-2045(16)30122-X, indexed in Pubmed: 27480103.

56. Robert C, Karaszewska B, Schachter J, et al. Three-year estimate of overall survival in COMBI-v, a randomized phase 3 study evaluating first-line dabrafenib $(D)+$ trametinib $(T)$ in patients (pts) with unresectable or metastatic BRAF V600E/K-mutant cutaneous melanoma. Annals of Oncology. 2016; 27: vi575, doi: 10.1093/annonc/mdw435.37.

57. Dummer R, Ascierto PA, Gogas HJ, et al. Encorafenib plus binimetinib versus vemurafenib or encorafenib in patients with BRAF-mutan melanoma (COLUMBUS): a multicentre, open-label, randomised phase 3 trial. Lancet Oncol. 2018; 19(5): 603-615, doi: 10.1016/S14702045(18)30142-6, indexed in Pubmed: 29573941.

58. Dummer R, Ascierto PA, Gogas HJ, et al. Overall survival in patients with BRAF-mutant melanoma receiving encorafenib plus binimetinib versus vemurafenib or encorafenib (COLUMBUS): a multicentre, open-label, randomised, phase 3 trial. Lancet Oncol. 2018; 19(10): 1315-1327, doi: 10.1016/S1470-2045(18)30497-2, indexed in Pubmed: 30219628
59. Robert C, Grob JJ, Stroyakovskiy D, et al. Five-Year Outcomes with Dabrafenib plus Trametinib in Metastatic Melanoma. N Engl J Med. 2019; 381(7): 626-636, doi: 10.1056/NEJMoa1904059, indexed in Pubmed: 31166680

60. Schreuer M, Jansen Y, Planken S, et al. Combination of dabrafenib plus trametinib for BRAF and MEK inhibitor pretreated patients with advanced BRAF-mutant melanoma: an open-label, single arm, dual-centre, phase 2 clinical trial. Lancet Oncol. 2017; 18(4): 464-472, doi: 10.1016/S1470-2045(17)30171-7, indexed in Pubmed: 28268064 .

61. Valpione S, Carlino M, Mangana J, et al. Re-challenge with BRAF-directed treatment: A multi-institutional retrospective study. Journal of Clinical Oncology. 2017; 35(15_suppl): 9512-9512, doi: 10.1200/jco.2017.35.15 suppl.9512.

62. Cybulska-Stopa B, Rogala P, Czarnecka A, et al. BRAF and MEK inhibitors rechallenge as effective treatment for patients with metastatic melanoma. Melanoma Research. 2020: 1, doi: $10.1097 / \mathrm{cmr} .0000000000000662$

63. Czarnecka AM, Teterycz P, Mariuk-Jarema A, et al. Treatment Sequencing and Clinical Outcomes in BRAF-Positive and BRAF-Negative Unresectable and Metastatic Melanoma Patients Treated with New Systemic Therapies in Routine Practice. Target Oncol. 2019; 14(6): 729-742, doi: 10.1007/s11523-019-00688-8, indexed in Pubmed: 31754963.

64. Guo J, Si Lu, Kong Y, et al. Phase II, open-label, single-arm trial of imatinib mesylate in patients with metastatic melanoma harboring c-Kit mutation or amplification. J Clin Oncol. 2011; 29(21): 2904-2909, doi: 10.1200/JCO.2010.33.9275, indexed in Pubmed: 21690468.

65. Jassem J, Duchnowska R, Kawecki A, et al. Badania kontrolne po leczeniu w najczęstszych nowotworach litych u dorosłych. Nowotwory. Journal of Oncology. 2014: 64(5): 415-435, doi: 10.5603/njo.2014.0070.

66. Rutkowski P, Lugowska I. Follow-up in melanoma patients. Memo. 2014; 7(2): 83-86, doi: 10.1007/s12254-014-0151-y, indexed in Pubmed: 25089158 